\title{
Herds on green meadows: the decarbonization of institutional portfolios
}

\author{
Lukas Benz ${ }^{1} \cdot$ Andrea Jacob $^{1} \cdot$ Stefan Paulus ${ }^{1} \cdot$ Marco Wilkens $^{1}[$
}

Revised: 13 December 2019 / Published online: 25 January 2020

(c) The Author(s) 2020

\begin{abstract}
We analyze an emerging sustainable trend in asset management: the decarbonization of institutional portfolios. By using broad institutional ownership data, we show that investors exhibit herding behavior in the sense of decarbonization. They are inclined to follow their own or other investors' buys in green stocks and sales in brown stocks over adjacent quarters. Beyond that, we find that Hedge Funds as well as Investment Advisors lead the herd by executing trades in the sense of decarbonization. This is in line with expectations that sophisticated investors, who integrate environmental aspects into their investment decision process, are able to attract imitators. For the aspired achievement of market-wide decarbonization, investors leading the herd should be encouraged to further decarbonize their portfolios in order to trigger follow-up trades.
\end{abstract}

Keywords Decarbonization · Institutional investors $\cdot$ Herding

JEL Classification G11 $\cdot \mathrm{G} 15 \cdot \mathrm{G} 23 \cdot \mathrm{M} 14$

\section{Introduction}

The combat of climate change has evolved as a global challenge for the entire society. We observe changing awareness and behavioral attitudes of society toward environmentalrelated issues. Besides, governments already take action or prepare to do so in order to mitigate the effects of climate change. In addition, the economy is in a state of transformation toward more environmental-friendly processes and activities.

The overarching political global target that represents climate change-induced action is the reduction of global warming to well below $2{ }^{\circ} \mathrm{C}$ above pre-industrial levels. However, not only politics and economics are concerned with environmental changes and transformations, but also the financial market. It has to adapt to changes and take a key role in the achievement of sustainable targets. For example, the EU Action Plan on Financing Sustainable Growth of the European Commission expects the financial industry to be

Marco Wilkens

marco.wilkens@wiwi.uni-augsburg.de

1 Chair of Finance and Banking, University of Augsburg, Universitaetsstr. 16, 86159 Augsburg, Germany a part of the solution toward a greener economy (European Commission 2018).

We analyze one of the emerging sustainable trends in asset management: the decarbonization of institutional portfolios. Our aim is to answer the question whether the decarbonization movement is observable in the financial market or whether engaging institutions are merely an exceptional phenomenon. For this purpose, we address different aspects of portfolio decarbonization using the largest institutional ownership dataset in literature so far and combine existing methodologies for herding measurement.

Since greenhouse gas emissions are one of the main causes of global warming, their reduction constitutes the main target function for politics and society to combat climate change and achieve the global $2{ }^{\circ} \mathrm{C}$ target. In its traditional form, decarbonization describes the action to divest from carbon-intensive ("brown") assets and invest in lowcarbon ("green") assets instead (PDC 2015). In recent times, it encompasses far more than greenhouse gas emissionsthe goal should be to align the portfolio with the climate economy of the future.

The decarbonization or divestment movement has already been initiated back in 2011, as students called for climate 
action in the management of university endowments. ${ }^{1}$ Since then, commitments to divest have continued to grow rapidly, also among institutional investors, such as insurers, pension funds, and sovereign wealth funds.

The Portfolio Decarbonization Coalition (PDC) is a driving force to increase popularity of portfolio decarbonization. Its overall aim is to "drive greenhouse gas emissions reductions by mobilizing a critical mass of institutional investors committed to gradually decarbonizing their portfolios" (PDC 2015). We base our research questions on the target setting of the PDC, since it encompasses all aspects of our research.

First, we associate the mobilization of a critical mass with herding behavior. We follow the definition of Sias (2004) who terms herding as institutional investors following each other into and out of the same securities over adjacent periods. Our first research question thus examines whether institutional investors in general demonstrate herding behavior. We show that they in fact do. To be more specific, investors tend to follow the trades of other investors rather than following their own. For this purpose, we use the most comprehensive ownership dataset so far applied in literature.

Second, we analyze whether herding behavior is also present in the context of decarbonization by developing a unique approach to unveil such a pattern. We define decarbonization herding as investors following their own or others' buy trades in green stocks and their own or others' sell trades in brown stocks, respectively. On the contrary, carbonization herding is defined as investors following their own or others' buy trades in brown stocks and sell trades in green stocks. Our results show that decarbonization herding exists and is of higher importance than carbonization herding (in the sense of a significant positive difference between decarbonization and carbonization herding).

Third, we examine which institutional investor groups follow or even promote this herding behavior. We expect that professionally managed funds, such as mutual funds and hedge funds, are promoting the overall decarbonization herding, since they are often bound to reputational concerns (Scharfstein and Stein 1990; Dasgupta et al. 2011). In addition, other investor groups might follow their lead, since they rely on the supposedly well-informed investment decisions of these professional portfolio managers. Besides, pension funds and insurance companies are bound to social norms (Hong and Kacperczyk 2009; Bolton and Kacperczyk 2019) and thus should follow the decarbonization trend. In fact, we find that Hedge Funds and Investments Advisors, which include Mutual Funds, Pension Funds, and Insurance

\footnotetext{
1 The most popular examples include Stanford University, Harvard University, Glasgow University, and Yale University, even though not all petitions were successful (see, e.g., Litterman 2015).
}

Companies, make up the largest part of herding and engage highly in decarbonization herding.

The remainder of the paper is structured as follows. "Background and related literature" section describes the theoretical foundation and summarizes the related literature. In "Sample construction and summary statistics" section, we explain the data. "Herding in the financial market" section presents the methodology used for the determination of overall herding in the financial market and the respective results, whereas "(De)carbonization herding" section introduces our measures of (de)carbonization herding and shows our results. "Robustness tests" section gives an overview of additional robustness tests, and "Conclusion" section concludes.

\section{Background and related literature}

Decarbonization describes the process of aligning one's portfolio with the low-carbon climate economy of the future. Recent research emphasizes the importance of climate risks for institutional investors. For example, Krueger et al. (forthcoming) conduct a survey with institutional investors and find that investors expect significant financial implications for firms due to climate risks. Some investors therefore try to reduce carbon footprints or stranded asset risks of their portfolios. In order to implement such decarbonization strategies, investors have to assess a firm's environmental performance. However, detailed information on a firm's carbon emissions, environmental strategy, and impact is rather scarce, since there does not exist a common disclosure standard on firm level yet. Investors thus have to take readily available information and base their decisions on their expectations on how the regulatory environment will change and how risk and return patterns will react to these changes. In this context, herding of institutional investors might arise to a number of intuitive reasons.

Bikhchandani and Sharma (2001) distinguish between intentional and spurious herding. They argue that "true" herding results from investors' obvious intent to copy the behavior of others neglecting their own available information. On the contrary, when investors take the same decisions without considering the actions of others due to the same decision problem and information set, the authors speak of spurious (unintentional) herding. In the context of decarbonization, it is plausible that investors follow the decisions of others intentionally, since they are aware that they face asymmetric information with respect to evaluating a firm's environmental performance and strategy. The scarcity of information might induce them to trust in decisions of others and follow them intentionally. However, as all investors probably have the same scarce information about firms and the regulatory environment at hand, investors' portfolio 
decisions can be the result of a common information set leading to unintentional herding behavior. Whether investors decarbonize their portfolios intentionally or unintentionally is difficult to analyze, since the real motive behind an investor's trade remains unknown. However, literature provides various explanations on why herding should occur-either intentionally or unintentionally. ${ }^{2}$ These explanations provide the theoretical foundation of our empirical analysis on decarbonization herding.

One of the simplest motives for herding is that investors follow trends. In recent times, we observe an increased awareness for sustainability in society as well as the call for financing more sustainable projects and stocks (e.g., the Fridays for future movement and the EU Action Plan on Financing Sustainable Growth). In turn, green stocks become more popular and brown stocks lose their standing inducing institutional investors to buy the former and sell the latter, i.e., to engage in decarbonization strategies. We expect this movement from a critical mass of investors, and hence, decarbonization herding should be observable in the financial market. Furthermore, due to its current relevance, we expect decarbonization herding to be more important than carbonization herding.

Besides, investors are dependent on retail flows. Since society strives for more sustainable and greener investments due to its increased sustainable awareness, retail flows into sustainable financial products automatically increase forcing investors to invest them in green products and divest from brown products. Again, investors might unintentionally show decarbonization herding behavior driven by retail flows.

Moreover, we observe informational cascades, i.e., investors do not make use of their own noisy signals, but infer information from previous investors' trades. Besides demand also the offer of sustainable products increases inducing institutional investors to intentionally follow the actions of others and promote decarbonization herding.

This might also be attractive to investors due to reputational issues: If investors face a reputational loss when acting differently from the herd, they are more inclined to follow others' trades. Increasing popularity of sustainability ratings for investment products and demand for disclosure of sustainability criteria in investment vehicles render this herding motive more than plausible. We expect especially norm constrained professionally managed funds such as pension funds and well-informed portfolio managers such as mutual funds and hedge funds to be driven by reputational motives to follow others' investment decisions and decarbonize their portfolios.

\footnotetext{
2 An overview of herding motives can be found, e.g., in Sias (2004) and Choi and Sias (2009).
}

Furthermore, investors are often attracted to stocks with certain characteristics (characteristic herding). If investors choose to invest green, they have a limited investment universe at hand, thus increasing the probability of unintentional herding for decarbonization.

Finally, investigative herding suggests that investors follow correlated signals at different times, which might reflect the process of incorporating information into prices. Climate change and respective policy action might involve risks for firm values (e.g., Dietz et al. 2016; Campiglio et al. 2018; Hong et al. 2019). These new risk sources have to be incorporated into market prices leading to spurious decarbonization herding, i.e., buy decisions for green stocks and sale decisions for brown stocks, respectively.

Recent political developments and a tightening regulatory environment, such as a binding implementation of the EU Action Plan on Financing Sustainable Growth, might intensify herding behavior in the sense of decarbonization of institutional investors. The EU Action Plan asks for a reorientation of capital flows toward a more sustainable economy, inclusion of environmental considerations of investors and asset managers into financial decision-making, and standards and labels for sustainable financial products, among others (European Commission 2018). Worldwide, governments ratify the Paris Agreement and commit to actively combat climate change. ${ }^{3}$ Facing these political developments, the motives for decarbonization herding seem more relevant than ever before.

So far, literature does not show any analyses based on the theoretical foundation of decarbonization herding described above. This paper closes this research gap and analyses the intersection between herding, institutional ownership, and decarbonization. We briefly summarize the findings of these different strands of literature below.

Lakonishok et al. (1992) develop a simultaneous herding measure on stock level. Their results point to the assumption that money managers do not herd at a large and meaningful way, but rather follow a variety of trading strategies. Grinblatt et al. (1995) and Wermers (1999) study herding behavior of mutual funds. In their baseline tests, they use the herding measure of Lakonishok et al. (1992); however, they do not find high levels of herding in mutual funds. Sias (2004) measures herding as the cross-sectional correlation between institutional demand of subsequent quarters instead of testing cross-sectional temporal dependence of trades within a period as in Lakonishok et al. (1992). The author finds a positive correlation between institutional demand over adjacent quarters. Furthermore, he shows that herding is attributable both to investors following their own last-quarter's trades and others' last-quarter's trades. Choi and Sias (2009) and

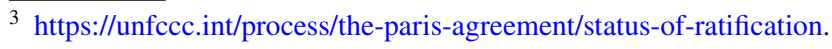


Celiker et al. (2015) use the Lakonishok et al. (1992) and the Sias (2004) methodology to answer the question whether institutional investors and mutual funds, respectively, follow each other into and out of the same industry. Both papers find strong evidence for industry herding. Popescu and $\mathrm{Xu}$ (2018) extend the methodology of Sias (2004) and compute measures for buy-leading and sell-leading of mutual funds. Lastly, Jiang and Verardo (2018) develop a herding measure on fund level that controls for investment styles and institutional preferences. We confirm results of previous studies and show that for our sample, herding is observable following the methodology of Sias (2004) and Popescu and Xu (2018).

The literature covering sustainability takes on various facets. The literature on divestment, however, is still scarce. In their survey of institutional investors, Krueger et al. (forthcoming) find out that institutional investors use a variety of approaches for climate risk management. In particular, investors analyze firms' carbon footprints and stranded asset risks, whereas some actively attempt to reduce these measures for their portfolio. Divesting problematic portfolio firms is also a practiced management technique. Trinks et al. (2017) analyze the financial costs of divestment for investor portfolios and find that divested portfolios do not underperform the unconstrained market portfolio. Davies and Van Wesep (2018) show in a quantification exercise of their economic model which consequences divestment campaigns have for investors and firms' managers. Boermans and Galema (2019) demonstrate that Dutch pension funds actively decarbonize their portfolios. They focus their analysis on pension funds, since these funds face divestment pressure from stakeholders.

Lastly, we present literature on the intersection of institutional ownership and sustainability considerations. Graves and Waddock (1994) find that institutional investors respond favorably to improvements in corporate social responsibility, i.e., institutional ownership measured by the number of institutions owning shares increases when corporate social performance improves. Likewise, Chava (2014) shows that fewer institutional investors hold shares of firms with environmental weaknesses. In addition, the author demonstrates that holdings from norm constrained institutions such as pension funds are significantly lower in firms with higher environmental concerns in latter time periods. This speaks for an increased environmental awareness of institutional investors. Hong and Kacperczyk (2009) focus on so-called "sin stocks" and show that sin stocks have a significantly lower ownership ratio. In turn, Bolton and Kacperczyk (2019) find out that insurance companies, investment advisors, and pension funds own less high-emission firms. Besides, Dyck et al. (2019) analyze the impact of institutional investors on the environmental and social performance of a firm. They show that institutional ownership positively impacts environmental and social performance.

With this paper, we contribute to the herding literature by focusing on the decarbonization of institutional portfolios. For this purpose, we combine three research areas-herding, institutional investors, and sustainability - to derive important insights into the sustainability trend in asset management. With our unique approach of measuring (de)carbonization herding, we answer the research question whether institutional investors tend to follow their own or others' trades that point to the decarbonization of portfolios. Our results confirm that herding in the financial market is driven by decarbonization trades. Thus, we demonstrate that institutional investors indeed tend to follow the decarbonization movement in the financial market and are inclined to pursue sustainable trends in asset management.

\section{Sample construction and summary statistics}

We obtain data from different databases. The ownership data is sourced from the Refinitiv Ownership and Profiles database. ${ }^{4}$ This is the most complete global shares database providing insight into the ownership structure of over 70,000 publicly traded equities in over 70 markets. The data are sourced from stock exchanges, regulatory bodies, institutions, and different financial reports, e.g., 13F filings. It covers different types of investors, e.g., Investment Advisors, Governmental Agencies, Foundations, and Individual Investors from all over the world. The database reports for each quarter the shares held and the respective market value of the investors' positions at the respective firms as well as the percentage held.

We run several filters to fit the ownership data to our needs. In order to prove herding behavior for investors, we need information on the trading activity of individual investors. We define an investor's trading activity as purchasing a stock if the percentage held increased over the quarter. In the opposite case, the investor's activity is identified as selling the stock. However, the ownership data obtained from Refinitiv contain the ownership structure of stocks and not trading data, which means that we obtain information on which shareholders hold the particular stock in their portfolio and to what extent. To fully determine the investor's trading activity, we complement our dataset to control for interim and final sales. We exclude all investors if they do not trade at least one stock over the observation period, and we exclude investors with less than eight quarters of ownership data.

\footnotetext{
${ }^{4}$ Formerly known as Thomson Reuters Ownership data.
} 
We categorize stocks as brown and green using ESG data of the Refinitiv $\mathrm{ESG}^{5}$ database. With information for over 7000 firms, this database covers the most important stocks traded on global stock markets and therefore serves as a good proxy for the worldwide investment universe. Refinitiv uses over 400 ESG metrics and assesses firms on the basis of their sustainable use of resources, emissions, environmental production processes, human rights, and management structures. It is currently perceived as one of the best available sources for firms' environmental, social, and governance information. Due to the fact that we want to analyze (de)carbonization herding, we focus only on one of the three pillars, namely the environmental pillar score (E-score). Hereby, firms are ranked according to their resource usage, emission reduction efforts, and their degree of environmental innovation. Every year, we calculate the median E-Score of all firms and identify firms with an E-Score greater (smaller) than the median as green (brown), which leads to half of the stocks being classified as green and brown, respectively, each year.

We do not solely focus on carbon emissions to identify brown and green stocks for many reasons. First of all, data availability on carbon emissions is rather scarce. Furthermore, investors choose different strategies to actually decarbonize their portfolios (e.g., exclusionary criteria, carbon footprints, and engagement; PDC 2017). This makes it difficult to measure decarbonization herding based on one specific stock criterion. Besides, due to recent developments, financial market participants demand forward-looking assessments for sustainability. PDC (2017) emphasizes that investors have to systematically determine and consider climate-related risks and opportunities. Hence, "portfolio decarbonization refers to systematic efforts by investors to align their investment portfolios with the goals of a lowcarbon economy" (PDC 2017). This subsumes not only the reduction of the carbon footprint, but also, e.g., an increased investment in renewable energies and capital withdrawal from high energy consumption activities (PDC 2017). For this reason, we choose to label firms as brown or green based on the environmental pillar score, since it comprises a more forward-looking assessment of sustainability and a wider scope than just carbon emissions. ${ }^{6}$

Table 1 reports the descriptive statistics for our sample. From January 2002 to September 2017, we observe 5669 distinct firms, for which we were able to obtain ownership data on a quarterly basis and for which ESG data are

\footnotetext{
5 Previously known as Thomson Reuters ASSET4 ESG.

6 According to Refinitiv, the environmental score "reflects how well a company uses best management practices to avoid environmental risks and capitalize on environmental opportunities" (Thomson Reuters 2015). The score thus is appropriate for our needs to identify stocks for portfolio decarbonization.
}

available. The number of firms in this study increases each year until it reaches its peak in 2016 with 5064 stocks. Each quarter, we identify an investor as a buyer if her ownership in the respective stock increases compared to the last quarter and as a seller if it decreases. The number of identified trades increases nearly sevenfold from 2002 to $2016 .{ }^{7}$ The most trades can be observed in 2016 with around 4.5 million trades. Over our sample period, we identify slightly more buys than sales with an average buy to overall trades ratio of around $53 \%$, which is in line with existing literature (Wermers 1999; Choi and Sias 2009).

Due to the fact that we distinguish between green and brown stocks, we also consider the respective buy and sell ratios of these two stock groups. The buy and sell ratio of brown stocks are on average higher than the buy and sell ratio of green stocks. The decarbonization ratio (sum of the buy ratio of green stocks and the sell ratio of brown stocks) and the carbonization ratio (sum of the buy ratio of brown stocks and the sell ratio of green stocks) do not exhibit large differences with the decarbonization ratio being slightly higher on average.

Table 2 contains statistics about the investor types. For the 5669 firms, we obtain ownership information for over 137,976 investors and we classify each of them into one of eight investor types. ${ }^{8}$ To the best of our knowledge, the ownership dataset in this study is, so far, the largest one used to study herding behavior. Just like the number of stocks, the number of investors increases over the sample period with a maximum of 77,640 unique investors in 2016 as shown in Panel A. The number of observed investors differs greatly between the different investor types. Only around 30 investors are identified as Foundations, while up to 60,000 (78\%) of the observed investors are identified as Individual Investors. But, more interesting than the absolute number of investors is the number of trades that these groups execute (Panel B). Although Individual Investors make up the largest share of the observed investors, they do not trade as much as the group of Hedge Funds and Investment Advisors. The most trades are executed by Investment Advisors with nearly 2.5 million trades in 2016.

\footnotetext{
7 The number of trades in 2017 decreases due to the fact that we obtain ownership data only until September 2017. We are therefore missing one quarter in 2017.

8 The original investor types reported by Refinitiv are more granular, but we group similar investor types together for the sake of clarity. Endowment Funds are included in Foundations, Research Firms are included in Banks and Trusts, Private Equity includes Holding Companies and Venture Capital Firms, and Investment Advisors include Mutual Funds, Pension Funds, and Insurance Companies.
} 


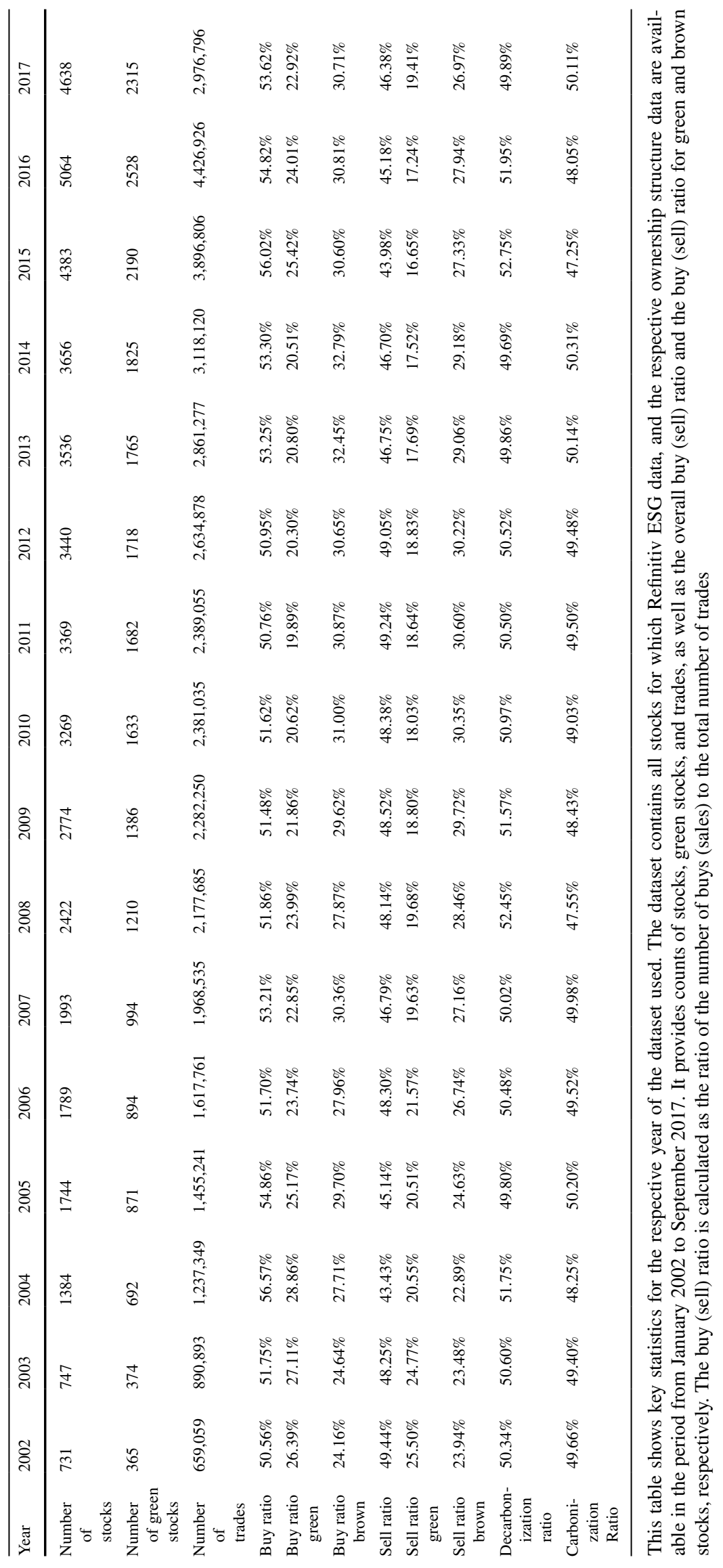

站- 


\begin{tabular}{|c|c|c|c|c|c|c|c|c|c|c|c|c|c|c|c|}
\hline & Ð & $\infty$ & 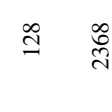 & 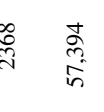 & 总 & 壳 & id & 盖 & 孚 总 & $\begin{array}{l}\infty \\
\stackrel{8}{0} \\
\stackrel{0}{-1}\end{array}$ & $\begin{array}{l}\vec{D} \\
0 \\
\overrightarrow{7} \\
\overrightarrow{7}\end{array}$ & $\begin{array}{l}\infty \\
\text { ते } \\
\text { है }\end{array}$ & $\begin{array}{l}\overrightarrow{\bar{g}} \\
\bar{c} \\
\bar{c}\end{array}$ & 萦 & 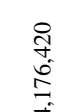 \\
\hline & 声 & $\infty$ & $\begin{array}{ll}\mathscr{8} \\
\stackrel{d}{d}\end{array}$ & 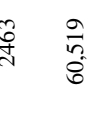 & 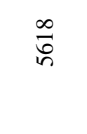 & 品 & 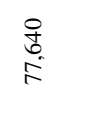 & 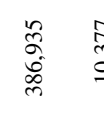 & 点 & 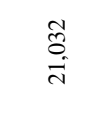 & 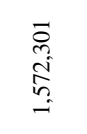 & di & 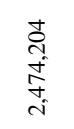 & 㤐 & $\begin{array}{l}m \\
\vdots \\
0 \\
y \\
+ \\
f \\
f\end{array}$ \\
\hline aे & 宇 & $\approx$ & $\stackrel{\infty}{\stackrel{0}{9}}$ & 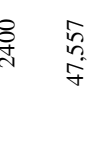 & 茥 & 寺 & 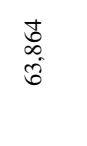 & 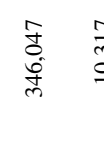 & 虔 & 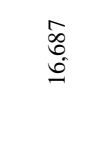 & $\begin{array}{l}\infty \\
\infty \\
\infty \\
\infty \\
-\infty \\
-1\end{array}$ & $\begin{array}{l}\text { aे } \\
\text { in }\end{array}$ & $\begin{array}{l}0 \\
0 \\
0 \\
0 \\
0 \\
0 \\
i\end{array}$ & ڤ్రి & 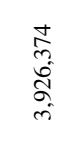 \\
\hline & 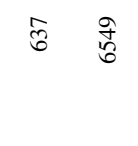 & 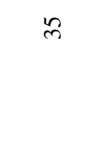 & $\stackrel{\infty}{\rightarrow} \quad \stackrel{n}{m}$ & 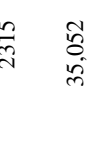 & हె & 志 & $\begin{array}{l}\stackrel{m}{\vec{t}} \\
\stackrel{\dot{m}}{n}\end{array}$ & 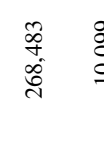 & 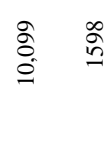 & $\begin{array}{l}\text { 释 } \\
\text { f }\end{array}$ & $\begin{array}{l}\tilde{Z} \\
\hat{\sigma} \\
\dot{0}\end{array}$ & 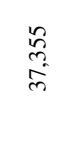 & $\begin{array}{l}\stackrel{8}{8} \\
\stackrel{+}{ \pm} \\
= \\
=\end{array}$ & तี & $\begin{array}{l}\text { t. } \\
\text { o. } \\
0 \\
0 \\
0 \\
m\end{array}$ \\
\hline 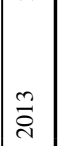 & 总总 & $m$ & 志 & 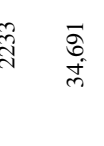 & $\vec{i}$ & ర్ల & $\begin{array}{l}\vec{b} \\
\stackrel{g}{q}\end{array}$ & 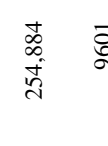 & స్ర్ & 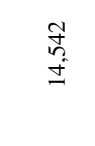 & $\begin{array}{l}\frac{m}{2} \\
\frac{0}{2} \\
0 \\
0\end{array}$ & $\begin{array}{l}\ddot{B} \\
\stackrel{0}{0} \\
\stackrel{\infty}{0}\end{array}$ & 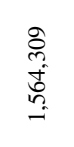 & $\stackrel{m}{\infty}$ & $\begin{array}{l}\overrightarrow{\mathrm{N}} \\
0 \\
0 \\
0 \\
\text { i }\end{array}$ \\
\hline & $8 \%$ & $\infty$ & తి $\stackrel{\infty}{\stackrel{\infty}{\sim}}$ & 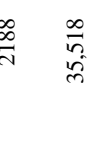 & 导 & $\underset{\mathrm{D}}{\mathbf{t}}$ & $\begin{array}{l}\tilde{\sigma} \\
\tilde{\sigma} \\
\dot{\sigma}\end{array}$ & 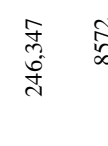 & 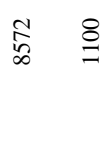 & $\underset{\substack{0 \\
\stackrel{0}{0}}}{0}$ & $\begin{array}{l}\text { 吕 } \\
\text { aे }\end{array}$ & $\begin{array}{l}\text { Fे } \\
\text { 它 } \\
\text { ch }\end{array}$ & 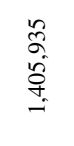 & $\underset{d}{\frac{\pi}{\sim}}$ & 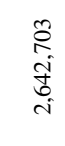 \\
\hline 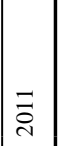 & 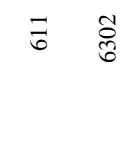 & in & $\stackrel{ }{\simeq} \quad$ & 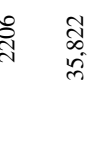 & 拳 & f & $\frac{q}{q}$ & $\begin{array}{ll}0 & 0 \\
0 & 0 \\
0 & 0 \\
0 & 0\end{array}$ & 寗 & $\begin{array}{l}\infty \\
\infty \\
\infty \\
\\
\end{array}$ & $\begin{array}{l}\text { 品 } \\
\stackrel{0}{\infty} \\
\infty\end{array}$ & $\begin{array}{c}\vec{E} \\
\dot{0} \\
\infty\end{array}$ & 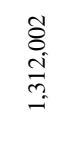 & $\stackrel{\infty}{\sim}$ & 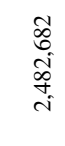 \\
\hline 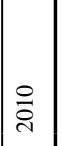 & కี & m & I & 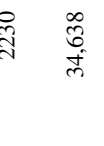 & 类 & 启 & $\begin{array}{l}\stackrel{8}{8} \\
\text { ó } \\
\text { of }\end{array}$ & $\begin{array}{ll}\infty & 0 \\
\infty & 5 \\
& \vdots \\
& 5\end{array}$ & \begin{tabular}{ll}
0 \\
\multirow{2}{*}{}
\end{tabular} & $\stackrel{\mathbb{\Xi}}{=}$ & 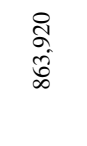 & 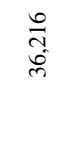 & 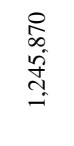 & 兽 & 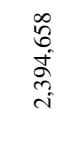 \\
\hline छे & E) & $\approx$ & $\equiv \quad \mathbb{N}$ & 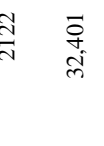 & 粱 & ฉั & $\begin{array}{l}\infty \\
\infty \\
\infty \\
f\end{array}$ & $\begin{array}{l}0 \\
\text { ป̀ } \\
\text { ปn }\end{array}$ & ఫ્ळે \& & $\stackrel{\infty}{\stackrel{\infty}{\Xi}}$ & 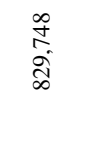 & 俤 & 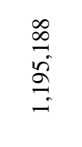 & 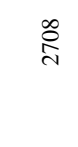 & 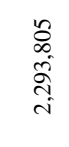 \\
\hline 离 & d్ $\quad \underset{f}{F}$ & 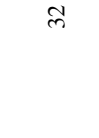 & $\stackrel{\Xi}{\stackrel{ \pm}{*}}$ & 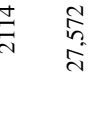 & 㐫 & : & $\begin{array}{l}\vec{\infty} \\
\bar{\delta} \\
\bar{\delta}\end{array}$ & 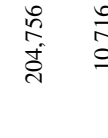 & 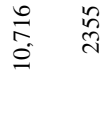 & ఫ్ & 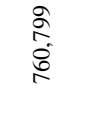 & $\begin{array}{l}n \\
\text { in } \\
0 \\
0 \\
0\end{array}$ & $\begin{array}{l}\stackrel{E}{E} \\
\stackrel{d}{=}\end{array}$ & $\overrightarrow{\mathrm{g}}$ & $\begin{array}{c}\infty \\
0 \\
0 \\
\infty \\
0 \\
i \\
i\end{array}$ \\
\hline 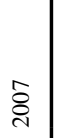 & 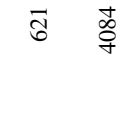 & $\bar{m}$ & $\because \quad \frac{1}{0}$ & 竎 & $\stackrel{\circ}{\stackrel{乛}{7}}$ & ల్ & $\begin{array}{l}\infty \\
\infty \\
\infty \\
ల\end{array}$ & 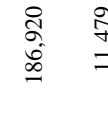 & 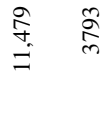 & 农 & $\begin{array}{l}\text { वे } \\
\text { हैं }\end{array}$ & $\begin{array}{l}\text { 品 } \\
\text { d̦ }\end{array}$ & 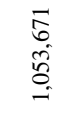 & $\overrightarrow{\mathrm{C}}$ & $\begin{array}{l}m \\
\bar{a} \\
0 \\
5 \\
- \\
-1\end{array}$ \\
\hline ڤั & 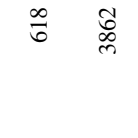 & i & $\vec{n} \quad \stackrel{+}{\Delta}$ & 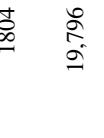 & 拿 & $\stackrel{\circ}{\bar{N}}$ & $\begin{array}{l}\text { o } \\
\text { 离 } \\
\text { d. }\end{array}$ & 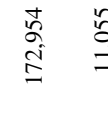 & 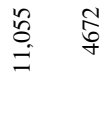 & $\stackrel{\circ}{\frac{9}{g}}$ & $\frac{b}{\frac{b}{f}}$ & 吉 & 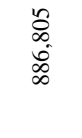 & 离 & 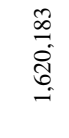 \\
\hline है & 竞 & $\infty$ & F & 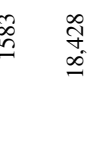 & 总 & $\stackrel{\leftrightarrow}{\sim}$ & 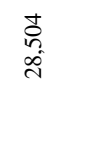 & 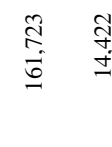 & $\begin{array}{ll}\underset{7}{7} & \bar{g} \\
\end{array}$ & 卓 & רิ & $\stackrel{9}{\overrightarrow{7}}$ & 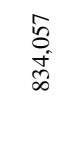 & 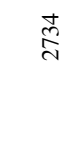 & 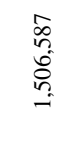 \\
\hline & 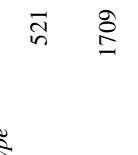 & $\vec{m}$ & F $\stackrel{\circ}{\stackrel{9}{g}}$ & 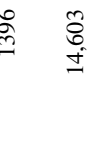 & 点 & $\vec{\Xi}$ & $\begin{array}{l}\text { Sa } \\
\text { ga }\end{array}$ & 昰 & $\vec{~} \overline{0}$ & $\stackrel{\bar{m}}{m}$ & $\begin{array}{l}\vec{m} \\
\text { s. } \\
\dot{m}\end{array}$ & 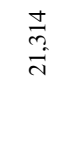 & 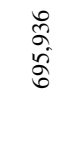 & $\stackrel{n}{E}$ & 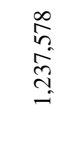 \\
\hline ڤ్ & 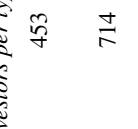 & $\bar{\lambda}$ & $\vec{m} \quad \stackrel{\partial}{=}$ & \&. & 营 & 8 & 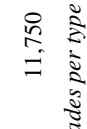 & $\begin{array}{ll}\infty & 0 \\
0 & 0 \\
\infty & 0\end{array}$ & 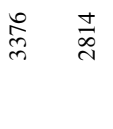 & 范 & 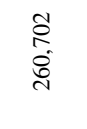 & 总 & $\begin{array}{l}\frac{ \pm}{D} \\
\hat{D} \\
\dot{n}\end{array}$ & $\vec{\infty}$ & $\begin{array}{l}\infty \\
\stackrel{\infty}{+} \\
\stackrel{\infty}{\infty}\end{array}$ \\
\hline ठ్రి & 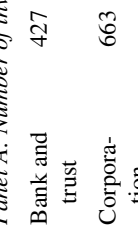 & $\bar{a}$ & 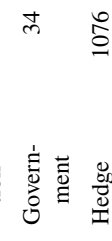 & : & 号 & n & 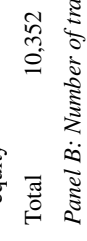 & 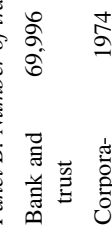 & 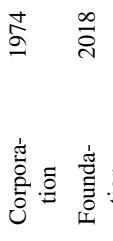 & 5 & 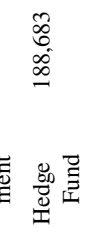 & స్ & 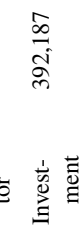 & 导 & $\begin{array}{l}\overline{8} \\
8 \\
0\end{array}$ \\
\hline
\end{tabular}




\section{Herding in the financial market}

At first, we analyze in how far the financial market exhibits herding behavior. For this purpose, we combine the methodologies of Sias (2004) and Popescu and Xu (2018). In this way, we do not only connect different measurement methods to derive more detailed insights into herding behavior, but
Herding can derive from different sources. Therefore, Sias (2004) decomposes the beta coefficient into two sources of herding: investors following their own trades, i.e., they trade the same stock in the same direction in the following period, and other investors following the trade of the investor, i.e., in the following period, another investor trades the same stock in the same direction.

$$
\begin{aligned}
\beta_{t}= & \rho\left(\Delta_{i, t+1}, \Delta_{i, t}\right) \\
= & \frac{1}{\left(I_{t}-1\right) \sigma\left(\mathrm{br}_{i, t}\right) \sigma\left(\mathrm{br}_{i, t+1}\right)} \sum_{i=1}^{I_{t}} \sum_{n=1}^{N_{i, t}} \frac{\left(D_{n, i, t+1}-\overline{\mathrm{br}_{t+1}}\right)\left(D_{n, i, t}-\overline{\mathrm{br}_{t}}\right)}{N_{i, t} N_{i, t+1}} \\
& +\frac{1}{\left(I_{t}-1\right) \sigma\left(\mathrm{br}_{i, t}\right) \sigma\left(\mathrm{br}_{i, t+1}\right)} \sum_{i=1}^{I_{t}} \sum_{n=1}^{N_{i, t}} \sum_{m=1, m \neq n}^{N_{i, t+1}} \frac{\left(D_{m, i, t+1}-\overline{\mathrm{br}_{t+1}}\right)\left(D_{n, i, t}-\overline{\mathrm{br}_{t}}\right)}{N_{i, t} N_{i, t+1}}
\end{aligned}
$$

also do so by using the largest institutional ownership dataset so far in the literature.

For each stock in each quarter, we calculate the buy ratio, defined as the number of all purchases in that stock divided by all trades in that specific stock.

$$
\text { br }_{i, t}=\frac{\text { \#of buys }_{i, t}}{\text { \#of buys }_{i, t}+\text { \#f sells }_{i, t}}
$$

As seen in Table 1, the average buy ratio over all stocks over the whole sample period is approximately $53 \%$.

Following Sias (2004), we further calculate the standardized buy ratio of stock $i$ as

$\Delta_{i, t}=\frac{\mathrm{br}_{i, t}-\overline{\mathrm{br}_{t}}}{\sigma\left(\mathrm{br}_{t}\right)}$

where $\overline{\mathrm{br}_{t}}$ is the cross-sectional average of the buy ratio in quarter $t$ over all stocks $i$ and $\sigma\left(\mathrm{br}_{t}\right)$ is the cross-sectional standard deviation in quarter $t$ over all stocks $i$.

As in Sias (2004), we define herding as investors following themselves or each other into or out of the same stock over consecutive quarters. In order to prove herding behavior, the cross-sectional correlation of the institutional investors' demand for a share between two consecutive quarters is calculated. This cross-sectional correlation is equivalent to the coefficient from the regression of the standardized institutional demand for share $i$ in quarter $t+1$ on the standardized institutional demand for share $i$ in quarter $t$.

$\Delta_{i, t+1}=\beta_{t} \Delta_{i, t}+\varepsilon_{i, t}$

Due to the standardized factors, the constant is zero and the coefficient $\beta_{t}$ simplifies to

$\beta_{t}=\rho\left(\Delta_{i, t+1}, \Delta_{i, t}\right)$ where $N_{i, t}$ is the number of investors trading stock $i$ in quarter $t$ and $D_{n, i, t}$ is a dummy variable that equals one (zero) if investor $n$ buys (sells) the stock $i$ in quarter $t$. Likewise, $N_{i, t+1}$ is the number of investors trading stock $i$ in quarter $t+1$, $D_{n, i, t+1}$ is a dummy variable that equals one (zero) if investor $n$ buys (sells) the stock $i$ in quarter $t+1$, and $D_{m, i, t+1}$ is a dummy variable that equals one (zero) if investor $m(m \neq n)$ buys (sells) the stock $i$ in quarter $t+1 . I_{t}$ is the overall number of stocks which are traded in quarter $t$. The first part of Eq. (5) represents the part of the correlation that results from investors following their own trades in consecutive quarters, i.e., buying (selling) a share in $t$ and buying (selling) it again in $t+1$. We expect this term to be positive if investor trading is persistent over time, i.e., investors trade the same stock in the same direction a quarter later. If investors tend to avoid executing the same trades as before, the term becomes negative. If there is no systematic correlation between the trading activities of investors in two consecutive quarters, the sum for this quarter becomes zero. The second part of the crosssectional correlation in Eq. (5) results from other investors following the trades of the respective investor. The same expectations regarding the direction of the correlation apply here. This method therefore helps to answer the question whether investors follow themselves or others when trading the same stocks in the following quarter.

Table 3 Panel A reports the average herding coefficients over 62 cross-sectional regressions and the associated t-statistics. The average cross-sectional correlation of the lagged and the current institutional demand is significantly positive with a value of 0.3203 which is in line with existing literature, such as Sias (2004), Choi and Sias (2009), and Celiker et al. (2015). This illustrates clearly that on average the financial market shows herding behavior.

In a next step, we decompose the herding measure into different sources of herding. One arises from investors following their own trades, which we call self-herding, and the 
Table 3 Herding in the financial market

Panel A: Tests for overall herding

\begin{tabular}{|c|c|c|c|c|c|c|c|c|c|}
\hline & \multicolumn{2}{|c|}{ Average herding $(\beta)$} & \multicolumn{2}{|c|}{ Self-herding } & \multicolumn{2}{|r|}{$\%$} & \multicolumn{2}{|c|}{ Following-herding } & $\%$ \\
\hline Securities with $\geq 1$ traders & \multicolumn{2}{|c|}{$\begin{array}{c}0.3203 \\
\left(34.16^{* * *}\right)\end{array}$} & \multicolumn{2}{|c|}{$\begin{array}{c}0.0161 \\
(8.21 * * *)\end{array}$} & \multicolumn{2}{|r|}{$5.03 \%$} & \multicolumn{2}{|c|}{$\begin{array}{c}0.3042 \\
(34.09 * * *)\end{array}$} & $94.97 \%$ \\
\hline Securities with $\geq 50$ traders & \multicolumn{2}{|c|}{$\begin{array}{c}0.3228 \\
(34.28 * * *)\end{array}$} & \multicolumn{2}{|c|}{$\begin{array}{c}0.0153 \\
(8.14 * * *)\end{array}$} & \multicolumn{2}{|r|}{$4.74 \%$} & \multicolumn{2}{|c|}{$\begin{array}{c}0.3074 \\
(34.43 * * *)\end{array}$} & $95.26 \%$ \\
\hline Securities with $\geq 100$ traders & \multicolumn{2}{|c|}{$\begin{array}{c}0.3237 \\
\left(33.10^{* * *}\right)\end{array}$} & \multicolumn{2}{|c|}{$\begin{array}{c}0.0132 \\
\left(7.98^{* * *}\right)\end{array}$} & \multicolumn{2}{|r|}{$4.09 \%$} & \multicolumn{2}{|c|}{$\begin{array}{c}0.3104 \\
(32.80 * * *)\end{array}$} & $95.91 \%$ \\
\hline \multicolumn{10}{|c|}{ Panel B: Tests for buy and sell herding } \\
\hline & $\begin{array}{c}\text { Average } \\
\text { herding }(\beta)\end{array}$ & $\begin{array}{c}\text { Buy self- } \\
\text { herding }\end{array}$ & $\%$ & $\begin{array}{l}\text { Sell self- } \\
\text { herding }\end{array}$ & $\%$ & $\begin{array}{l}\text { Buy follow- } \\
\text { ing-herding }\end{array}$ & $\%$ & $\begin{array}{c}\text { Sell } \\
\text { following- } \\
\text { herding }\end{array}$ & $\%$ \\
\hline Securities with $\geq 1$ traders & $\begin{array}{c}0.3203 \\
\left(34.16^{* * *}\right)\end{array}$ & $\begin{array}{l}0.0032 \\
\left(1.97^{*}\right)\end{array}$ & $1.00 \%$ & $\begin{array}{c}0.0129 \\
(8.52 * * *)\end{array}$ & $4.03 \%$ & $\begin{array}{c}0.1420 \\
(30.17 * * *)\end{array}$ & $44.32 \%$ & $\begin{array}{c}0.1623 \\
(31.44 * * *)\end{array}$ & $50.66 \%$ \\
\hline Securities with $\geq 50$ traders & $\begin{array}{c}0.3228 \\
(34.28 * * *)\end{array}$ & $\begin{array}{l}0.0029 \\
\left(1.87^{*}\right)\end{array}$ & $0.89 \%$ & $\begin{array}{c}0.0124 \\
(9.11 * * *)\end{array}$ & $3.86 \%$ & $\begin{array}{c}0.1435 \\
(30.43 * * *)\end{array}$ & $44.46 \%$ & $\begin{array}{c}0.1639 \\
(31.98 * * *)\end{array}$ & $50.80 \%$ \\
\hline Securities with $\geq 100$ traders & $\begin{array}{c}0.3237 \\
\left(33.10^{* * *}\right)\end{array}$ & $\begin{array}{c}0.0018 \\
(1.24)\end{array}$ & $0.56 \%$ & $\begin{array}{c}0.0114 \\
(9.00 * * *)\end{array}$ & $3.53 \%$ & $\begin{array}{c}0.1454 \\
(29.25 * * *)\end{array}$ & $44.91 \%$ & $\begin{array}{c}0.1651 \\
(30.87 * * *)\end{array}$ & $50.99 \%$ \\
\hline
\end{tabular}

This table shows tests for herding measures for securities with equal or more than 1, 50, and 100 traders, respectively, for the sample period. Panel A calculates the overall average herding measure as well as the self- and following-herding using the methodology described in Sias (2004). Panel B additionally decomposes herding in buy and sell herding in the sense of Popescu and Xu (2018). The columns labeled as "\%" indicates the share of the respective herding source on the average herding measure. Statistical significance is measured by two-sided $t$ tests and $*$, **, and $* * *$ denote statistical significance at the $10 \%, 5 \%$, and $1 \%$ level, respectively. T-statistics are shown in parentheses

other from other investors following the trades of the respective investor, which we refer to as following-herding. Both components make a significant contribution to the average herding measure. Around 5\% of average herding results from self-herding, which is in line with the hypothesis that institutional investors split bigger investments into several trades over some period to minimize their price impact (Chakravarty 2001). However, as seen in Panel A, most of the herding, nearly $95 \%$, derives from following-herding. Choi and Sias (2009) have already shown this pattern when taking aggregated stock industries into account rather than individual stocks. A slightly increased herding measure and the same pattern also occur when only stocks traded by more than 50 or 100 investors are considered.

Some investors are limited in their actions due to shortselling or liquidity constraints (Wylie 2005). Thus, the herding resulting from a stock sale might be limited in comparison to herding resulting from a buy of the same stock. In the spirit of Popescu and $\mathrm{Xu}$ (2018), we further extend the Sias (2004) model and decompose Eq. (5) to compute the portion of self-herding or following-herding triggered by investors' buys or sales. In this way, we eliminate the possibility of confounding effects between buy and sale trades. 


$$
\begin{aligned}
\beta_{t}= & \rho\left(\Delta_{i, t+1}, \Delta_{i, t}\right) \\
= & \frac{1}{\left(I_{t}-1\right) \sigma\left(\mathrm{br}_{i, t}\right) \sigma\left(\mathrm{br}_{i, t+1}\right)} \sum_{i=1}^{I_{t}} \sum_{n=1}^{N_{i, t}} \frac{\left(D_{n, i, t+1}-\overline{\mathrm{br}_{t+1}}\right)\left(1-\overline{\mathrm{br}_{t}}\right)}{N_{i, t} N_{i, t+1}} \\
& +\frac{1}{\left(I_{t}-1\right) \sigma\left(\mathrm{br}_{i, t}\right) \sigma\left(\mathrm{br}_{i, t+1}\right)} \sum_{i=1}^{I_{t}} \sum_{n=1}^{N_{i, t}} \frac{\left(D_{n, i, t+1}-\overline{\mathrm{br}_{t+1}}\right)\left(0-\overline{\mathrm{br}_{t}}\right)}{N_{i, t} N_{i, t+1}} \\
& +\frac{1}{\left(I_{t}-1\right) \sigma\left(\mathrm{br}_{i, t}\right) \sigma\left(\mathrm{br}_{i, t+1}\right)} \sum_{i=1}^{I_{t}} \sum_{n=1}^{N_{i, t}} \sum_{m=1, m \neq n}^{N_{i, t+1}} \frac{\left(D_{m, i, t+1}-\overline{\mathrm{br}_{t+1}}\right)\left(1-\overline{\mathrm{br}_{t}}\right)}{N_{i, t} N_{i, t+1}} \\
& +\frac{1}{\left(I_{t}-1\right) \sigma\left(\mathrm{br}_{i, t}\right) \sigma\left(\mathrm{br}_{i, t+1}\right)} \sum_{i=1}^{I_{t}} \sum_{n=1}^{N_{i, t}} \sum_{m=1, m \neq n}^{N_{i, t+1}} \frac{\left(D_{m, i, t+1}-\overline{\mathrm{br}_{t+1}}\right)\left(0-\overline{\mathrm{br}_{t}}\right)}{N_{i, t} N_{i, t+1}}
\end{aligned}
$$

Equation (6) is a more detailed version of Eq. (5), whereby a distinction is made between herding resulting from a purchase or a sale by assigning a strict value to $D_{n, i, t}\left(D_{m, i, t}\right)$, i.e., 1 for purchase and 0 for sale for the trade of investor $n(m)$ of stock $i$ in quarter $t$. This decomposition enables us to show whether investors mainly exhibit self- or following-herding when the triggering trade is a buy or a sale.

In Panel B of Table 3, the sum of self-herding resulting from buys (0.0032) and sales $(0.0129)$ has to be equal to the general self-herding measure (0.0161) from Panel A, whereas the same applies to following-herding. Furthermore, the sum of all four components corresponds to the general average herding coefficient of 0.3203 . The results show that herding is triggered by both buys and sales. In unreported analyses, we show that the differences between the buy and sell herding for self-herding as well as for following-herding are statistically significant. More important, all four herding sources have a significant share on the overall herding measure. The largest portion of herding is triggered by the sale of a stock $(54.69 \%)$, whereby for the most part (50.66\%), this sale functioned as a signal for other investors to sell this stock as well in the following quarter. The results are consistent with existing studies and are robust when considering only stocks that are traded by more than 50 or 100 investors.

\section{(De)carbonization herding}

In the last section, we have shown that the financial market approximated by our large dataset exhibits herding behavior. This section develops the methodology derived before further to break down the herding measure in (de)carbonization herding.

Henceforth, we distinguish between self-herding and following-herding induced by purchases or sales of green and brown stocks, i.e., we differentiate whether the triggering buy or sale in quarter $t$ takes place in a green or a brown stock. For this purpose, we extend Eq. (6) once again. ${ }^{9}$ To answer our main research question, we differentiate between herding in the sense of decarbonization and carbonization.

We define decarbonization herding as the behavior of investors to buy a green stock which was recently bought by the investor herself or some other investor, as well as to sell a brown stock which was recently sold by the investor herself or some other investor. Thus, we capture whether triggering trades in the spirit of decarbonization cause the same investor or other investors to decarbonize their portfolios as well in the subsequent quarter and thus induce herding behavior.

The (de)carbonization herding measures are computed as follows.

decarbonization selfherding , $_{t}$

$$
\begin{aligned}
= & \frac{1}{\left(I_{t}-1\right) \sigma\left(\mathrm{br}_{i, t}\right) \sigma\left(\mathrm{br}_{i, t+1}\right)} \sum_{i=1}^{I_{t, \text { green }}} \sum_{n=1}^{N_{i, t}} \frac{\left(D_{n, i, t+1}-\overline{\mathrm{br}_{t+1}}\right)\left(1-\overline{\mathrm{br}_{t}}\right)}{N_{i, t} N_{i, t+1}} \\
& +\frac{1}{\left(I_{t}-1\right) \sigma\left(\mathrm{br}_{i, t}\right) \sigma\left(\mathrm{br}_{i, t+1}\right)} \sum_{i=1}^{I_{t, b r o w n}} \sum_{n=1}^{N_{i, t}} \frac{\left(D_{n, i, t+1}-\overline{\mathrm{br}_{t+1}}\right)\left(0-\overline{\mathrm{br}_{t}}\right)}{N_{i, t} N_{i, t+1}}
\end{aligned}
$$

\footnotetext{
${ }^{9}$ For a more detailed derivation, see the "Appendix".
} 
decarbonization followingherding ${ }_{t}$

$$
\begin{aligned}
= & \frac{1}{\left(I_{t}-1\right) \sigma\left(\mathrm{br}_{i, t}\right) \sigma\left(\mathrm{br}_{i, t+1}\right)} \sum_{i=1}^{I_{t, g r e e n}} \sum_{n=1}^{N_{i, t}} \sum_{m=1, m \neq n}^{N_{i, t+1}} \frac{\left(D_{m, i, t+1}-\overline{\mathrm{br}_{t+1}}\right)\left(1-\overline{\mathrm{br}_{t}}\right)}{N_{i, t} N_{i, t+1}} \\
& +\frac{1}{\left(I_{t}-1\right) \sigma\left(\mathrm{br}_{i, t}\right) \sigma\left(\mathrm{br}_{i, t+1}\right)} \sum_{i=1}^{I_{t, b r o w n}} \sum_{n=1}^{N_{i, t}} \sum_{m=1, m \neq n}^{N_{i, t+1}} \frac{\left(D_{m, i, t+1}-\overline{\mathrm{br}_{t+1}}\right)\left(0-\overline{\mathrm{br}_{t}}\right)}{N_{i, t} N_{i, t+1}}
\end{aligned}
$$

where $I_{t, \text { green }}$ and $I_{t, \text { brown }}$ indicate the number of stocks identified as green or brown.

The decarbonization following-herding equals the sum of the following-herding resulting from the buy of a green stock and the following-herding resulting from the sale of a brown stock. In contrast, herding in the sense of carbonization would be the exact opposite, i.e., the portion of the herding measure resulting from the same investor or other investors increasing their shares in a brown stock by following the respective investor's buy of a brown stock and decreasing their shares in a green stock following the sale of the green stock.

carbonization selfherding ${ }_{t}$

$$
\begin{aligned}
= & \frac{1}{\left(I_{t}-1\right) \sigma\left(\mathrm{br}_{i, t}\right) \sigma\left(\mathrm{br}_{i, t+1}\right)} \sum_{i=1}^{I_{t, b \text { rown }}} \sum_{n=1}^{N_{i, t}} \frac{\left(D_{n, i, t+1}-\overline{\mathrm{br}_{t+1}}\right)\left(1-\overline{\mathrm{br}_{t}}\right)}{N_{i, t} N_{i, t+1}} \\
& +\frac{1}{\left(I_{t}-1\right) \sigma\left(\mathrm{br}_{i, t}\right) \sigma\left(\mathrm{br}_{i, t+1}\right)} \sum_{i=1}^{I_{t, \text { gren }}} \sum_{n=1} \frac{\left(D_{n, i, t+1}-\overline{\mathrm{br}_{t+1}}\right)\left(0-\overline{\mathrm{br}_{t}}\right)}{N_{i, t} N_{i, t+1}}
\end{aligned}
$$

process compared to carbonization herding. The majority of the herding, over $96 \%$, results from the decarbonization behavior of investors. The biggest share of this herding measure (0.3002) results from following-herding. It seems that investors are more likely to buy green shares which were bought by other investors and to sell brown shares which were sold by other investors in the preceding quarter. We can therefore confirm our hypothesis that for the global acting investors observed in this study, there exists herding in terms of the aspired decarbonization process. ${ }^{10}$ In contrast, decarbonization self-herding $(0.0087)$ as well as the carbonization following-herding (0.0040) do not have a significant influence on the overall herding measure over the observed 62 quarters.

To get a better understanding about herding in our sample, Fig. 1 plots the decarbonization and carbonization herding measures for self- and following-herding. As already seen in Table 4, self-herding for decarbonization as well as carbonization is rather small and on average close to zero over time. In contrast, following-herding is much more

carbonization followingherding ${ }_{t}$

$$
\begin{aligned}
= & \frac{1}{\left(I_{t}-1\right) \sigma\left(\mathrm{br}_{i, t}\right) \sigma\left(\mathrm{br}_{i, t+1}\right)} \sum_{i=1}^{I_{t, \text { brown }}} \sum_{n=1}^{N_{i, t}} \sum_{m=1, m \neq n}^{N_{i, t+1}} \frac{\left(D_{m, i, t+1}-\overline{\mathrm{br}_{t+1}}\right)\left(1-\overline{\mathrm{br}_{t}}\right)}{N_{i, t} N_{i, t+1}} \\
& +\frac{1}{\left(I_{t}-1\right) \sigma\left(\mathrm{br}_{i, t}\right) \sigma\left(\mathrm{br}_{i, t+1}\right)} \sum_{i=1}^{I_{t, \text { green }}} \sum_{n=1}^{N_{i, t}} \sum_{m=1, m \neq n}^{N_{i, t+1}} \frac{\left(D_{m, i, t+1}-\overline{\mathrm{br}_{t+1}}\right)\left(0-\overline{\mathrm{br}_{t}}\right)}{N_{i, t} N_{i, t+1}}
\end{aligned}
$$

where $I_{t, \text { green }}$ and $I_{t, \text { brown }}$ indicate the number of stocks identified as green or brown.

As shown before, we observe significant herding behavior in the financial market. If there was no preference for either green or brown stocks when following own trades or trades of other investors, the four carbonization and decarbonization herding measures introduced above should have approximately the same value. We show that this is not the case.

Table 4 shows the average of the different herding sources. The sum of the four (de)carbonization herding measures equals the overall herding measure of the financial market (0.3203). As we can see, there is a significant difference in the herding we attribute to the decarbonization volatile and reveals some interesting interrelations over time. Even though the average carbonization followingherding is close to $0(0.0040)$, it is negative most of the time from 2006 to 2012. As defined before, carbonization herding covers following trades on sales of green stocks and purchases of brown stocks. Thus, a negative carbonization herding coefficient relates to investors who tend to buy green

\footnotetext{
${ }^{10}$ In unreported analysis, we show that, as expected, the overall herding measure is driven by the largest investors in terms of assets under management. Nevertheless, we find for every investor size that trades in the sense of decarbonization trigger more follow-up trades than trades in the sense of carbonization. Results are available upon request from the authors.
} 
Table 4 (De)carbonization herding
Fig. 1 Herding behavior over time. This figure plots the (de) carbonization self- and following-herding measures over the sample period from January 2002 to September 2017. The herding measures are calculated as described in the text

\begin{tabular}{|c|c|c|c|c|c|}
\hline & Average herding $(\beta)$ & $\begin{array}{l}\text { Average herding } \\
(\beta) \text { per group }\end{array}$ & $\%$ & Self-herding & Following-herding \\
\hline Decarbonization & $\begin{array}{l}0.3203 \\
\left(34.16^{* * *}\right)\end{array}$ & $\begin{array}{l}0.3089 \\
\left(15.36^{* * *}\right)\end{array}$ & 96.42 & $\begin{array}{l}0.0087 \\
(0.71)\end{array}$ & $\begin{array}{l}0.3002 \\
(6.04 * * *)\end{array}$ \\
\hline Carbonization & & $\begin{array}{l}0.0115 \\
(15.11 * * *)\end{array}$ & 3.58 & $\begin{array}{l}0.0074 \\
(6.32 * * *)\end{array}$ & $\begin{array}{l}0.0040 \\
(0.25)\end{array}$ \\
\hline Difference & & $\begin{array}{l}0.2974 \\
(8.45 * * *)\end{array}$ & & $\begin{array}{l}0.0012 \\
(0.70)\end{array}$ & $\begin{array}{l}0.2962 \\
(8.44 * * *)\end{array}$ \\
\hline
\end{tabular}

This table shows the decarbonization and carbonization herding measures as defined in the text for the sample period. The column labeled as "\%" indicates the share of the (de)carbonization measure on the average herding measure in the financial market. Statistical significance is measured by two-sided $t$ tests and $* * *$, and $* * *$ denote statistical significance at the $10 \%, 5 \%$, and $1 \%$ level, respectively. T-statistics are shown in parentheses

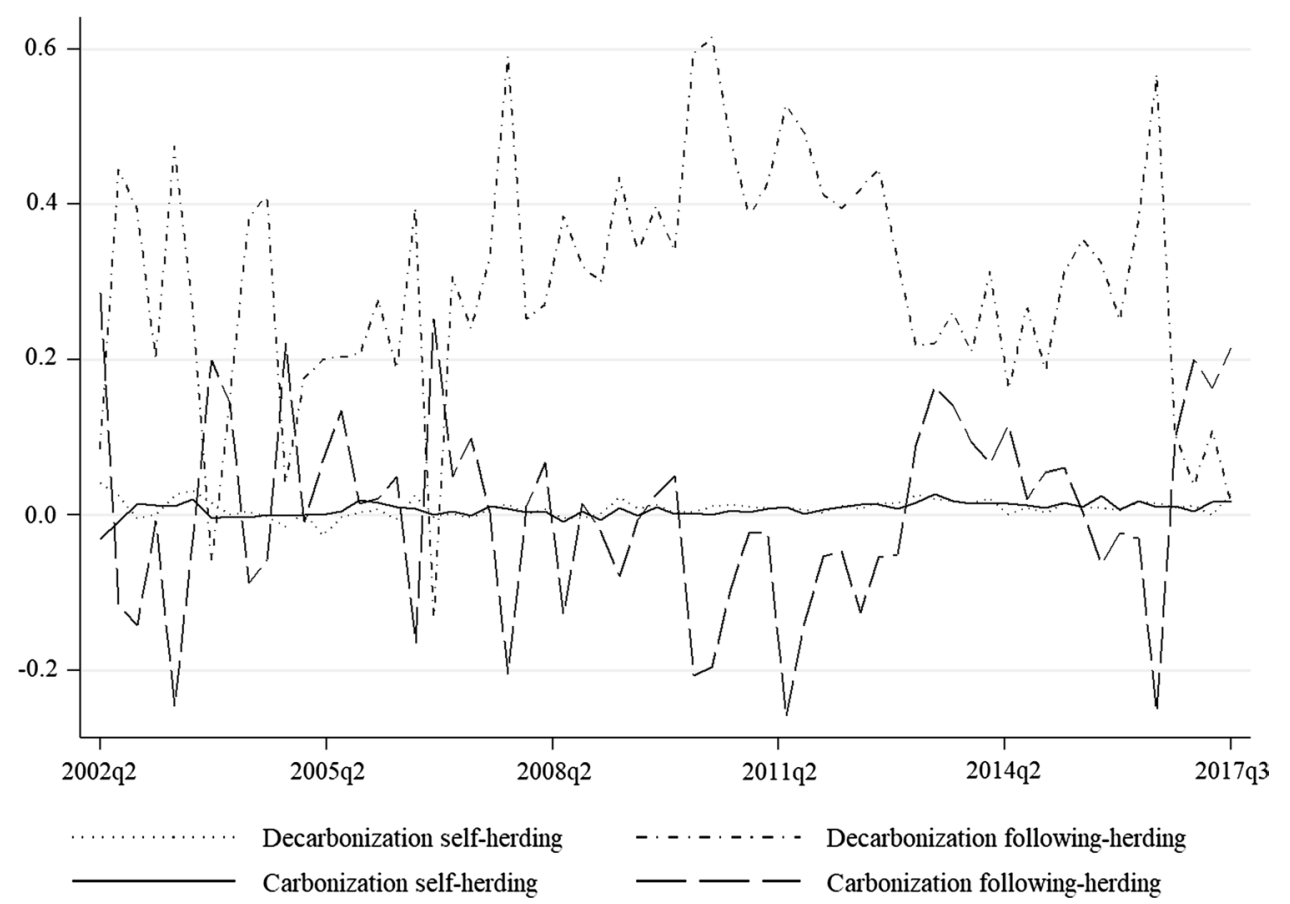

stocks that others have previously sold and sell brown stocks that other investors have previously bought. Therefore, the following investors decarbonize their portfolios. Taking this into account, the actual decarbonization effect is even greater than initially assumed. Until 2006, the differences between decarbonization and carbonization herding are not as clear-cut as in the following period. In 2006, the decarbonization following-herding leaps from -13 to $+30 \%$, and the decarbonization and carbonization following-herding diverge strongly from this moment on. We interpret this as a movement of investors who pay more attention to whether other investors decarbonize their portfolios and then follow this strategy. Around the years 2010 and 2011, we can see the highest decarbonization following-herding with over $60 \%$ and the most negative carbonization followingherding around $-26 \%$. From the third to the last quarter of 2016, we notice an interesting pattern. Decarbonization following-herding drops significantly, whereas carbonization following-herding increases over the level of decarbonization following-herding. In this time period, investors rather followed others' buys in brown stocks and sales in green stocks. The events happening in this time period provide a valid explanation for this pattern. In November 2016, Donald Trump won the Presidential Election. Trump often stated that he does not believe in climate change and finally decided to withdraw from the Paris Agreement (Pompeo 2019). In December 2016, he nominated Scott Pruitt, an opponent to climate change combat, as head of the US Environmental Protection Agency (Springer Nature 2016). During the same time, the 2016 United Nations Climate Change Conference in Marrakesh led to a great disappointment among climate change campaigners (Worley 2016). Those events 
might have influenced expectations of institutional investors on future developments of the regulatory environment concerning climate change action, who in turn revised their decarbonization investment decisions, leading to decreased herding following trades in the spirit of decarbonization.

So far, we have proven that there is a general herding behavior in the financial market and moreover that investors pay attention to whether other investors decarbonize their portfolios. With our next analysis, we try to find out which investor type leads this decarbonization movement, which investor type carries out the buys and sales, which follows itself, and which is followed in the consecutive quarter by other investors. We therefore calculate the proportion of the overall herding attributable to one specific type of investor. We then calculate for each investor type the share of decarbonization and carbonization herding as we have shown in Table 4 for the entire sample of investors. The sum of all individual parts across investor types must be the same as the overall herding measure from the beginning $(0.3203)$.

For each type, we can confirm that the proportion of decarbonization herding is significantly higher than the one of carbonization herding. The only exception is Foundations, where we only find an insignificant but still positive difference. The difference is mostly driven by investors following other investors rather than themselves. A substantial part of overall herding is driven by the trades of Individual Investors $(5.10 \%)$ and Banks and Trusts $(4.65 \%)$. The trades of Investment Advisors and Hedge Funds trigger the major part of overall herding in the financial market $(53.08 \% / 34.43 \%)$, which is not surprising due to the high number of trades executed by these two investor types. $51.98 \%$ of overall herding is driven by triggering trades of Investment Advisors in the spirit of decarbonization. This means, if an Investment Advisor buys a green stock or sells a brown stock, it is more likely that another investor or the Advisor herself will follow her than if the Advisor traded exactly the opposite way in the first place.

To further test which investor type demonstrates the most decarbonization herding, we redo the analyses above, but now we consider the aggregated investor types rather than the underlying individual investors. Therefore, we consider every investor type as a single investment portfolio and aggregate the shares held of each stock across all investors from one type. By doing this, we consider whether the aggregated group of investors buys or sells the respective stock as well as account for different numbers of trades of the various investor types.

Table 6 Panel A shows a pattern similar to Table 3 Panel $\mathrm{B}$, where we can see the overall herding measure as well as the differentiation between herding induced by buys or sales. Due to the aggregation on investor type level, the overall level of herding is lower (0.0636) compared to the previous herding measure on investor level (0.3203). Besides, the proportion of self-herding is much larger than before. $32.73 \%$ of herding results from an investor type selling the same stock over consecutive quarters, therefore splitting its sales over consecutive quarters. This can be regarded as intra-group herding (herding within an investor type). The overall following-herding now accounts for only $44.89 \%$, whereas on investor level about $94.98 \%$ of the overall herding measure is determined by following-herding. Investor type self-herding accounts for $55.11 \%$, thus being more important for overall herding than following-herding. This leads to the conclusion that a lot of investors trade the same stocks the same direction over consecutive quarters as investors of the same investor type, e.g., Investment Advisors tend to follow the trades of other Investment Advisors.

Panel B is similar to Table 5 and shows the breakdown of the investor type level herding in terms of decarbonization and carbonization. Similar to before, for each type, except for Governments, a significant larger proportion of overall herding results from decarbonization trades. Hedge Funds (36.62\%) and Investment Advisors (33.03\%) still account for the largest part of overall herding; however, the share of Investment Advisors is lower than before (33.03 vs. 53.08\%). In turn, the percentage of the other investor types, especially the proportions of Banks and Trusts as well as Individual Investors, triggers more follow-up trades compared to the investor level measure in Table 5. When only taking selfherding into account, we find that Individual Investors as well as Private Equity firms are more likely to follow trades in the sense of carbonization rather than decarbonization. For following-herding, we find the same pattern as before, with herding in the sense of decarbonization being more pronounced.

The results in Tables 5 and 6 are in line with expectations on which investor type might be more inclined not only in herding behavior but also in portfolio decarbonization. Only a small part of decarbonization herding is driven by trades of Private Equity firms $(0.22 \%$ and $3.72 \%$, respectively). This is in line with Crifo et al. (2015) who state that sustainable firms are not particularly attractive for Private Equity investors compared to others. Hence, investment decisions are possibly not driven by the motive of portfolio decarbonization. The investment decisions of Governments $(0.56 \%$ or $6.15 \%$ of decarbonizaton herding) and Corporations $(2.20 \%$ and $4.48 \%$ ) are often linked to a strongly investor-specific purpose, which is only of a limited imitative nature. Governments hold large shares in basic industries and the transportation industry (Bortolotti and Faccio 2009) and thus might be rather inclined to retain control in certain firms building stable and long-term ownership. Thus, herding is not a relevant strategy for governmental purposes. In the case of Corporations, the investor-specific purpose is reflected in the intention to enter into strategic alliances according to their specific business needs (Allen and Phillips 2000). 
Table 5 (De)carbonization herding per investor type

\begin{tabular}{|c|c|c|c|c|c|}
\hline & $\begin{array}{l}\text { Average herding } \\
(\beta) \text { per group }\end{array}$ & $\%$ & Self-herding & Following-herding & Average herding $(\beta)$ \\
\hline \multicolumn{6}{|l|}{ Bank and trust } \\
\hline Decarbonization & 0.0127 & 3.97 & -0.0003 & 0.0131 & \\
\hline Carbonization & 0.0022 & 0.68 & 0.0002 & 0.0019 & \\
\hline Difference & $0.0106^{* * *}$ & & $-0.0006^{* * *}$ & $0.0111 * * *$ & \\
\hline \multicolumn{6}{|l|}{ Corporation } \\
\hline Decarbonization & 0.0071 & 2.20 & 0.0002 & 0.0069 & \\
\hline Carbonization & -0.0017 & -0.53 & 0.0000 & -0.0016 & \\
\hline Difference & $0.0087 * * *$ & & $0.0003 * *$ & $0.0085^{* * *}$ & \\
\hline \multicolumn{6}{|l|}{ Foundation } \\
\hline Decarbonization & 0.0002 & 0.06 & 0.0000 & 0.0002 & \\
\hline Carbonization & 0.0001 & 0.03 & 0.0000 & 0.0001 & \\
\hline Difference & 0.0001 & & $0.0000 * * *$ & 0.0001 & \\
\hline \multicolumn{6}{|l|}{ Government } \\
\hline Decarbonization & 0.0018 & 0.56 & -0.0001 & 0.0019 & \\
\hline Carbonization & 0.0007 & 0.21 & -0.0001 & 0.0008 & $0.3203\left(34.16^{* * *}\right)$ \\
\hline Difference & $0.0011 *$ & & 0.0000 & $0.0011^{*}$ & \\
\hline \multicolumn{6}{|l|}{ Hedge fund } \\
\hline Decarbonization & 0.1013 & 31.63 & 0.0043 & 0.0971 & \\
\hline Carbonization & 0.0090 & 2.80 & 0.0037 & 0.0053 & \\
\hline Difference & $0.0924 * * *$ & & 0.0006 & $0.0918 * * *$ & \\
\hline \multicolumn{6}{|l|}{ Individual investor } \\
\hline Decarbonization & 0.0186 & 5.79 & 0.0016 & 0.0169 & \\
\hline Carbonization & -0.0022 & -0.69 & -0.0002 & -0.0020 & \\
\hline Difference & $0.0208 * * *$ & & $0.0018 * *$ & $0.0190 * * *$ & \\
\hline \multicolumn{6}{|l|}{ Investment advisor } \\
\hline Decarbonization & 0.1665 & 51.98 & 0.0030 & 0.1635 & \\
\hline Carbonization & 0.0035 & 1.10 & 0.0038 & -0.0003 & \\
\hline Difference & $0.1630 * * *$ & & -0.0008 & $0.1638^{* * *}$ & \\
\hline \multicolumn{6}{|l|}{ Private equity } \\
\hline Decarbonization & 0.0007 & 0.22 & 0.0000 & 0.0007 & \\
\hline Carbonization & -0.0001 & -0.02 & 0.0000 & -0.0001 & \\
\hline Difference & $0.0008 * * *$ & & 0.0000 & $0.0008 * * *$ & \\
\hline
\end{tabular}

The table shows the average (de)carbonization herding measures per investor type in the sample period. The calculation of the herding measures follows the description in the text. Investors are grouped into investor types as described in the text. For comparison purposes, the overall average herding measure is shown as well. The column labeled as "\%" indicates the share of the (de)carbonization herding on the average herding measure. The rows denoted as "Difference" are the differences between the decarbonization and the carbonization measure per investor type. Statistical significance is measured by two-sided $t$ tests and $*, * *$, and $* * *$ denote statistical significance at the $10 \%, 5 \%$, and $1 \%$ level, respectively

As a main result, Investment Advisors seem to promote decarbonization herding besides Hedge Funds. This result confirms the hypothesis that Mutual Funds and especially Hedge Funds are considered as well-informed portfolio managers inducing other investors to follow their lead (Eichengreen et al. 1998). The reason for the strong herding movement after their trades could lie in the anticipatory ability of these sophisticated investors to recognize capital market trends such as decarbonization. The original motivation of the leading portfolio managers does not necessarily have to be based on noble intentions with the aim of acting in a sustainable way, but could also be based on profiting from expected price movements of green or brown stocks. In addition, professional money managers are exceptionally prone to reputational herding due to career concerns (Scharfstein and Stein 1990; Dasgupta et al. 2011) and certain types such as pension funds and insurance companies are bound to social norms (Hong and Kacperczyk 2009; Bolton and Kacperczyk 2019). All of these motives trigger a large herding movement for these investor types. 
Table 6 Investor type herding

Panel A: Tests for investor type overall herding

\begin{tabular}{|c|c|c|c|c|c|c|c|c|}
\hline Average herding $(\beta)$ & Buy self-herding & $\%$ & Sell self-herding & $\%$ & $\begin{array}{l}\text { Buy follow- } \\
\text { ing-herding }\end{array}$ & $\%$ & $\begin{array}{l}\text { Sell follow- } \\
\text { ing-herding }\end{array}$ & $\%$ \\
\hline $\begin{array}{l}0.0636 \\
(12.01 * * *)\end{array}$ & $\begin{array}{c}0.0142 \\
\left(7.45^{* * *}\right)\end{array}$ & $22.38 \%$ & $\begin{array}{c}0.0208 \\
(11.15 * * *)\end{array}$ & $32.73 \%$ & $\begin{array}{c}0.0141 \\
(6.68 * * *)\end{array}$ & $22.17 \%$ & $\begin{array}{c}0.0144 \\
\left(4.56^{* * *}\right)\end{array}$ & $22.72 \%$ \\
\hline
\end{tabular}

Panel B: (De)carbonization herding of investor types

\begin{tabular}{|c|c|c|c|c|c|}
\hline & $\begin{array}{l}\text { Average herding }(\beta) \\
\text { per group }\end{array}$ & $\%$ & Self-herding & Following-herding & Average herding $(\beta)$ \\
\hline \multicolumn{6}{|l|}{ Bank and trust } \\
\hline Decarbonization & 0.0091 & $14.33 \%$ & 0.0023 & 0.0068 & \\
\hline Carbonization & -0.0019 & $-3.03 \%$ & -0.0021 & 0.0002 & \\
\hline Difference & $0.0110 * * *$ & & $0.0044 * * *$ & $0.0066^{* *}$ & \\
\hline \multicolumn{6}{|l|}{ Corporation } \\
\hline Decarbonization & 0.0028 & $4.48 \%$ & 0.0002 & 0.0027 & \\
\hline Carbonization & -0.0050 & $-7.87 \%$ & -0.0002 & -0.0048 & \\
\hline Difference & $0.0078 * * *$ & & 0.0004 & $0.0075^{* * *}$ & \\
\hline \multicolumn{6}{|l|}{ Foundation } \\
\hline Decarbonization & 0.0023 & $3.64 \%$ & 0.0007 & 0.0016 & \\
\hline Carbonization & -0.0003 & $-0.50 \%$ & 0.0001 & -0.0004 & \\
\hline Difference & $0.0026 * * *$ & & 0.0006 & $0.0020 * * *$ & \\
\hline \multicolumn{6}{|l|}{ Government } \\
\hline Decarbonization & 0.0039 & $6.15 \%$ & 0.0025 & 0.0015 & \\
\hline Carbonization & 0.0017 & $2.68 \%$ & 0.0013 & 0.0004 & $0.0636(12.01 * * *)$ \\
\hline Difference & 0.0022 & & $0.0012 * *$ & 0.0011 & \\
\hline \multicolumn{6}{|l|}{ Hedge fund } \\
\hline Decarbonization & 0.0192 & $30.22 \%$ & 0.0086 & 0.0106 & \\
\hline Carbonization & 0.0041 & $6.40 \%$ & 0.0059 & -0.0018 & \\
\hline Difference & $0.0151 * * *$ & & $0.0027 * *$ & $0.0124 * * *$ & \\
\hline \multicolumn{6}{|l|}{ Individual investor } \\
\hline Decarbonization & 0.0064 & $10.09 \%$ & 0.0011 & 0.0053 & \\
\hline Carbonization & -0.0003 & $-0.53 \%$ & 0.0022 & -0.0025 & \\
\hline Difference & $0.0068 * * *$ & & $-0.0010^{* *}$ & $0.0078 * * *$ & \\
\hline \multicolumn{6}{|l|}{ Investment advisor } \\
\hline Decarbonization & 0.0179 & $28.09 \%$ & 0.0083 & 0.0096 & \\
\hline Carbonization & 0.0031 & $4.94 \%$ & 0.0045 & -0.0014 & \\
\hline Difference & $0.0147 * * *$ & & $0.0038 * * *$ & $0.0109 * * *$ & \\
\hline \multicolumn{6}{|l|}{ Private equity } \\
\hline Decarbonization & 0.0024 & $3.72 \%$ & -0.0004 & 0.0027 & \\
\hline Carbonization & -0.0018 & $-2.83 \%$ & 0.0001 & -0.0019 & \\
\hline Difference & $0.0042 * * *$ & & $-0.0005^{* * *}$ & $0.0046^{* * *}$ & \\
\hline
\end{tabular}

This table shows herding measures on investor type level for the sample period. Investors are grouped into investor types as described in the text. Panel A calculates the overall herding measure on investor type level as in Sias (2004) and decomposes it in buy and sell herding following Popescu and Xu (2018). Panel B shows the (de)carbonization herding measures per investor type. The rows denoted as "Difference" are the differences between the decarbonization and carbonization herding measure per investor type. Columns labeled as "\%" indicate the share of the respective herding measure on the overall average herding measure. Statistical significance is based on two-sided $t$ tests and *, **, and *** denote significance at the $10 \%, 5 \%$, and $1 \%$ level, respectively. In Panel A, t-statistics are shown in parentheses

\section{Robustness tests}

We conduct several additional analyses to test the robustness of our results. First, we divide stocks in green and brown based on a static selection process. A stock is labeled as green if its average environmental score is above the median of all scores in the sample, and brown otherwise. Since we do not require stocks to have a complete time series of scores 
Table 7 (De)carbonization herding per market capitalization quintiles

\begin{tabular}{|c|c|c|c|c|c|}
\hline Market capitalization quintile & Average herding $(\beta)$ per group & $\%$ & Self-herding & Following-herding & Average herding $(\beta)$ \\
\hline \multicolumn{6}{|l|}{1 (small) } \\
\hline Decarbonization & 0.1607 & 84.82 & 0.0082 & 0.1526 & \multirow{3}{*}{$\begin{array}{c}0.1895 \\
\left(16.69^{* * *}\right)\end{array}$} \\
\hline Carbonization & 0.0288 & 15.18 & 0.0122 & 0.0165 & \\
\hline Difference & $0.1320 * * *$ & & $-0.0041 * *$ & $0.1360 * * *$ & \\
\hline \multicolumn{6}{|l|}{2} \\
\hline Decarbonization & 0.0756 & 62.79 & 0.0021 & 0.0734 & \multirow{3}{*}{$\begin{array}{c}0.12039 \\
(8.93 * * *)\end{array}$} \\
\hline Carbonization & 0.0448 & 37.21 & 0.0036 & 0.0412 & \\
\hline Difference & 0.0308 & & -0.0015 & 0.0323 & \\
\hline \multicolumn{6}{|l|}{3} \\
\hline Decarbonization & 0.1133 & 67.92 & 0.0005 & 0.1129 & \multirow{3}{*}{$\begin{array}{c}0.16685 \\
\left(13.49^{* * *}\right)\end{array}$} \\
\hline Carbonization & 0.0535 & 32.08 & 0.0040 & 0.0496 & \\
\hline Difference & $0.0598 *$ & & -0.0035 & $0.0633 * *$ & \\
\hline \multicolumn{6}{|l|}{4} \\
\hline Decarbonization & 0.1218 & 64.81 & 0.0027 & 0.1191 & \multirow{3}{*}{$\begin{array}{c}0.18792 \\
(16.12 * * *)\end{array}$} \\
\hline Carbonization & 0.0661 & 35.19 & 0.0016 & 0.0645 & \\
\hline Difference & $0.0557 *$ & & 0.0011 & $0.0545^{*}$ & \\
\hline \multicolumn{6}{|l|}{5 (large) } \\
\hline Decarbonization & 0.1954 & 75.12 & 0.0075 & 0.1879 & \multirow{3}{*}{$\begin{array}{c}0.26011 \\
\left(21.85^{* * *}\right)\end{array}$} \\
\hline Carbonization & 0.0647 & 24.88 & 0.0015 & 0.0632 & \\
\hline Difference & $0.1307 * * *$ & & 0.0060 & $0.1247 * * *$ & \\
\hline
\end{tabular}

This table shows the decarbonization and carbonization herding measures as defined in the text for the sample period separately for different stock sizes. Each quarter we sort the observed stocks into quintiles based on their market capitalization. The column labeled as "\%" indicates the share of the (de)carbonization herding on the average herding measure. The rows denoted as "Difference" are the differences between the decarbonization and the carbonization measure per investor type. Statistical significance is measured by two-sided $t$ tests and *, **, and *** denote statistical significance at the $10 \%, 5 \%$, and $1 \%$ level, respectively

available, we assign the characteristic of greenness (brownness) to each stock from its existence on. Thus, we are able to extend our data sample to a time period from 2000 to 2017. All of our results remain economically the same. However, this procedure might lead to a look-ahead bias of the sustainability characteristic of a stock.

Second, we undertake a reclassification of the stocks. We classify the observed stocks not only into green and brown, but into three groups, i.e., green, brown, and neutral. Each year, we divide the stocks into tertiles according to their E-score. We classify the bottom third as brown, the middle third as neutral, and the top third as green. Herding resulting from trades in neutral stocks accounts for around onethird of the overall herding measure. Our main finding from Table 4, that herding in terms of decarbonization is more pronounced than herding in terms of carbonization, remains unchanged. ${ }^{11}$

Third, as shown by Sias (2004), herding behavior of institutional investors differs when considering different stock sizes measured by market capitalization. Depending on the

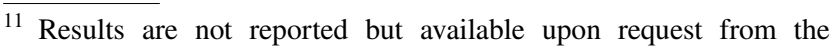
authors. herding motive used, the anticipated relation between stock size and herding measure differs. Informational cascades are more likely for small firms because investors might not make use of their own noisy signals, but infer information from previous investors' trades (Wermers 1999). Therefore, they overweight the information they gather from others and herding should be more pronounced within small stocks. On the contrary, if investigative herding is more pronounced among investors, i.e., investors trade on correlated signals, herding should be more pronounced in larger stocks (Sias 2004). Some could argue that our results regarding the more pronounced decarbonization herding than carbonization herding could be driven by stocks of a certain market capitalization. We follow Sias (2004) and sort the observed stocks each quarter into quintiles based on their market capitalization. We redo the research done above in Table 4 for each of the stock quintiles. Due to the use of standardized dependent and independent variables, the measured coefficients are comparable across quintiles.

As shown in Table 7, the average herding is more pronounced within large stocks compared to the herding in small stocks ( 0.26011 vs. 0.1895$)$, which contradicts the findings of Sias (2004). Following the reasoning of Sias (2004), we conclude that the herding observed in our 
research might result from the investigative motive. More interestingly, however, trades in the sense of decarbonization trigger more follow-up trades independent of the size of the traded security. Therefore, our results are not driven by size differences of the traded stocks.

Fourth, we substitute the environmental score with the overall ESG score to account for other aspects of sustainability. The results remain unchanged, even when including the social and governance dimension of sustainability. This mirrors the fact that the environmental score is highly correlated with the overall ESG score.

Last, we randomly assign stocks several times into the green and brown sample, respectively, regardless of their actual E-score. As expected, we do not find any statistically significant differences between decarbonization and carbonization herding. This verifies that the allocation of stocks based on the E-score does not follow a random criterion but is meaningful for the representation of (de)carbonization.

\section{Conclusion}

We analyze the presence of portfolio decarbonization in the financial market. For this purpose, we use the most comprehensive institutional ownership dataset in literature and combine herding measures from Sias (2004) and Popescu and $\mathrm{Xu}$ (2018). In the financial market, institutional investors tend to follow others rather than following their own trades regardless whether the triggering trade is a buy or a sale. In addition, we find that institutional investors engage in decarbonization herding. This means they follow their own or other investors' buy trades in green stocks and sell trades in brown stocks, respectively. It is noticeable that decarbonization herding is majorly triggered by following-herding, i.e., investors follow others in portfolio decarbonization. Across all investor types, decarbonization herding is greater and significantly different from carbonization herding. In specific, we show that especially Investment Advisors and Hedge Funds promote decarbonization herding.

Overall, institutional investors seem to be prone to the divestment movement in the sense that they are taking part in it. The motives for their herding behavior are manifold. Decarbonization herding might be intentional as well as spurious. Mutual Funds and Hedge Funds might engage in decarbonization herding due to reputational concerns. Others might follow their trades, since they infer superior information from the behavior of the more sophisticated investor group. Besides, Pension Funds and Insurance Companies are bound to social norms which lead them to invest in sustainable stocks. Another circumstance leading to decarbonization herding are uncertain information sets about firms' environmental strategies and the regulatory environment. Investors might act on the same available information and take similar investment decisions which eventually results in herding behavior. More empirical analyses on these herding motives are left to future research. Furthermore, the impact of decarbonization herding on stock prices of green and brown stocks, respectively, has yet to be determined.

We rather focus on an as-is description of the occurring phenomenon of portfolio decarbonization. In conclusion, for the aspired achievement of market-wide decarbonization, investors leading the herd should be encouraged to further decarbonize their portfolios in order to trigger follow-up trades by others. Our results point to the fact that climate change does not only become critically important in the financial market, but already has arrived in asset management.

Acknowledgments Open Access funding provided by Projekt DEAL.

Open Access This article is licensed under a Creative Commons Attribution 4.0 International License, which permits use, sharing, adaptation, distribution and reproduction in any medium or format, as long as you give appropriate credit to the original author(s) and the source, provide a link to the Creative Commons licence, and indicate if changes were made. The images or other third party material in this article are included in the article's Creative Commons licence, unless indicated otherwise in a credit line to the material. If material is not included in the article's Creative Commons licence and your intended use is not permitted by statutory regulation or exceeds the permitted use, you will need to obtain permission directly from the copyright holder. To view a copy of this licence, visit http://creativecommons.org/licenses/by/4.0/.

\section{Appendix}

In the following, we show the derivation of the decarbonization and carbonization herding measures in a more detailed way. Starting from Eq. (6), where we distinguish between self- and following-herding resulting from buys or sales separately, we further split up the equation. In each quarter $t$, we differentiate whether the triggering buy or sale takes place in a green or a brown stock. 


$$
\begin{aligned}
& \beta_{t}=\operatorname{cov}\left(\Delta_{i, t+1}, \Delta_{i, t}\right) \\
& =\frac{1}{\left(I_{t}-1\right) \sigma\left(\mathrm{br}_{i, t}\right) \sigma\left(\mathrm{br}_{i, t+1}\right)} \sum_{i=1}^{I_{t, \text { green }}} \sum_{n=1}^{N_{i, t}} \frac{\left(D_{n, i, t+1}-\overline{\mathrm{br}_{t+1}}\right)\left(1-\overline{\mathrm{br}_{t}}\right)}{N_{i, t} N_{i, t+1}} \\
& +\frac{1}{\left(I_{t}-1\right) \sigma\left(\mathrm{br}_{i, t}\right) \sigma\left(\mathrm{br}_{i, t+1}\right)} \sum_{i=1}^{I_{t, \text { brown }}} \sum_{n=1}^{N_{i, t}} \frac{\left(D_{n, i, t+1}-\overline{\mathrm{br}_{t+1}}\right)\left(1-\overline{\mathrm{br}_{t}}\right)}{N_{i, t} N_{i, t+1}} \\
& +\frac{1}{\left(I_{t}-1\right) \sigma\left(\mathrm{br}_{i, t}\right) \sigma\left(\mathrm{br}_{i, t+1}\right)} \sum_{i=1}^{I_{t, \mathrm{green}}} \sum_{n=1}^{N_{i, t}} \frac{\left(D_{n, i, t+1}-\overline{\mathrm{br}_{t+1}}\right)\left(0-\overline{\mathrm{br}_{t}}\right)}{N_{i, t} N_{i, t+1}} \\
& +\frac{1}{\left(I_{t}-1\right) \sigma\left(\mathrm{br}_{i, t}\right) \sigma\left(\mathrm{br}_{i, t+1}\right)} \sum_{i=1}^{I_{t, \text { brown }}} \sum_{n=1}^{N_{i, t}} \frac{\left(D_{n, i, t+1}-\overline{\mathrm{br}_{t+1}}\right)\left(0-\overline{\mathrm{br}_{t}}\right)}{N_{i, t} N_{i, t+1}} \\
& +\frac{1}{\left(I_{t}-1\right) \sigma\left(\mathrm{br}_{i, t}\right) \sigma\left(\mathrm{br}_{i, t+1}\right)} \sum_{i=1}^{I_{t, \text { green }}} \sum_{n=1}^{N_{i, t}} \sum_{m=1, m \neq n}^{N_{i, t+1}} \frac{\left(D_{m, i, t+1}-\overline{\mathrm{br}_{t+1}}\right)\left(1-\overline{\mathrm{br}_{t}}\right)}{N_{i, t} N_{i, t+1}} \\
& +\frac{1}{\left(I_{t}-1\right) \sigma\left(\mathrm{br}_{i, t}\right) \sigma\left(\mathrm{br}_{i, t+1}\right)} \sum_{i=1}^{I_{t, \text { brown }}} \sum_{n=1}^{N_{i, t}} \sum_{m=1, m \neq n}^{N_{i, t+1}} \frac{\left(D_{m, i, t+1}-\overline{\mathrm{br}_{t+1}}\right)\left(1-\overline{\mathrm{br}_{t}}\right)}{N_{i, t} N_{i, t+1}} \\
& +\frac{1}{\left(I_{t}-1\right) \sigma\left(\mathrm{br}_{i, t}\right) \sigma\left(\mathrm{br}_{i, t+1}\right)} \sum_{i=1}^{I_{t, \text { green }}} \sum_{n=1}^{N_{i, t}} \sum_{m=1, m \neq n}^{N_{i, t+1}} \frac{\left(D_{m, i, t+1}-\overline{\mathrm{br}_{t+1}}\right)\left(0-\overline{\mathrm{br}_{t}}\right)}{N_{i, t} N_{i, t+1}} \\
& +\frac{1}{\left(I_{t}-1\right) \sigma\left(\mathrm{br}_{i, t}\right) \sigma\left(\mathrm{br}_{i, t+1}\right)} \sum_{i=1}^{I_{t, \text { brown }}} \sum_{n=1}^{N_{i, t}} \sum_{m=1, m \neq n}^{N_{i, t+1}} \frac{\left(D_{m, i, t+1}-\overline{\mathrm{br}_{t+1}}\right)\left(0-\overline{\mathrm{br}_{t}}\right)}{N_{i, t} N_{i, t+1}}
\end{aligned}
$$

where $I_{t, \text { green }}$ and $I_{t \text {,brown }}$ indicate the number of stocks identified as green or brown in quarter $t$. It is clear that the sum of $I_{t, \text { green }}$ and $I_{t \text {,brown }}$ equals the overall number of traded stocks $I_{t}$ in this quarter. To calculate the portion of herding in the sense of decarbonization self-herding we then sum (a), following their own buy of a green stock, and (d), following their own sell of a brown stock. To calculate the portion of herding in the sense of carbonization self-herding, we then sum (b), following their own buy of a brown stock, and (c), following their own sale of a green stock. Likewise, decarbonization following-herding equals the sum of (e) and (h) and carbonization following-herding results from (f) plus (g).

\section{References}

Allen, J.W., and G.M. Phillips. 2000. Corporate Equity Ownership, Strategic Alliances, and Product Market Relationships. The Journal of Finance 55 (6): 2791-2815.

Bikhchandani, S., and S. Sharma. 2001. Herd Behavior in Financial Markets. IMF Staff Papers 47 (3): 279-310.

Boermans, M.A., and R. Galema. 2019. Are Pension Funds Actively Decarbonizing Their Portfolios? Ecological Economics 161: $50-60$.
Bolton, P., and M. Kacperczyk, 2019. Do Investors Care About Carbon Risk? Working Paper. https://doi.org/10.2139/ssrn.3398441.

Bortolotti, B., and M. Faccio. 2009. Government Control of Privatized Firms. The Review of Financial Studies 22 (8): 2907-2939.

Campiglio, E., Y. Dafermos, P. Monnin, J. Ryan-Collins, G. Schotten, and M. Tanaka. 2018. Climate Change Challenges for Central Banks and Financial Regulators. Nature Climate Change 8: 462-468.

Celiker, U., J. Chowdhury, and G. Sonaer. 2015. Do Mutual Funds Herd in Industries? Journal of Banking \& Finance 52: 1-16.

Chakravarty, S. 2001. Stealth-Trading: Which Traders' Trades Move Stock Prices? Journal of Financial Economics 61 (2): 289-307.

Chava, S. 2014. Environmental Externalities and Cost of Capital. Management Science 60 (9): 2223-2247.

Choi, N., and R.W. Sias. 2009. Institutional Industry Herding. Journal of Financial Economics 94 (3): 469-491.

Choi, D., Z. Gao, and W. Jiang. forthcoming. Attention to Global Warming. The Review of Financial Studies (in press).

Crifo, P., V.D. Forget, and S. Teyssier. 2015. The Price of Environmental, Social and Governance Practice Disclosure: An Experiment with Professional Private Equity Investors. Journal of Corporate Finance 30: 168-194.

Dasgupta, A., A. Prat, and M. Verardo. 2011. The Price Impact of Institutional Herding. The Review of Financial Studies 24 (3): 892-925.

Davies, S.W., and E.D. Van Wesep. 2018. The Unintended Consequences of Divestment. Journal of Financial Economics 128 (3): 558-575. 
Dietz, S., A. Bowen, C. Dixon, and P. Gradwell. 2016. 'Climate Value at Risk' of Global Financial Assets. Nature Climate Change 6: 676-679.

Dyck, A., K.V. Lins, L. Roth, and H.F. Wagner. 2019. Do Institutional Investors Drive Corporate Social Responsibility? International Evidence. Journal of Financial Economics 131 (3): 693-714.

Eichengreen, B., D. Mathieson, B. Chadha, A. Jansen, L. Kodres, and S. Sharma. 1998. Hedge Funds and Financial Market Dynamics. International Monetary Fund. Washington DC. Occasional Paper 166.

European Commission. 2018. Communication Form the Commission to the European Parliament, The European Council, the Council, the European Central Bank, the European Central Bank, the European Economic and Social Committee and the Committee of the Regions. Action Plan: Financing Sustainable Growth, 8 March 2018, COM(2018) 97 Final. https://eur-lex.europa.eu/legal -content/EN/TXT/PDF/?uri=CELEX:52018DC0097\&from=EN. Accessed 5 Sept 2019.

Graves, S.B., and S.A. Waddock. 1994. Institutional Owners and Corporate Social Performance. The Academy of Management Journal 37 (4): 1034-1046.

Grinblatt, M., S. Titman, and R. Wermers. 1995. Momentum Investment Strategies, Portfolio Performance, and Herding: A Study of Mutual Fund Behavior. The American Economic Review 85 (5): $1088-1105$.

Hong, H., and M. Kacperczyk. 2009. The Price of Sin: The Effects of Social Norms on Markets. Journal of Financial Economics 93 (1): $15-36$

Hong, H., F.W. Li, and J. Xu. 2019. Climate Risks and Market Efficiency. Journal of Econometrics 208 (1): 265-281.

Jiang, H., and M. Verardo. 2018. Does Herding Behavior Reveal Skill? An Analysis of Mutual Fund Performance. The Journal of Finance 73 (5): 2229-2269.

Krueger, P., Z. Sautner, and L.T. Starks. 2019. Swiss Finance Institute Research Paper No. 18-58; European Corporate Governance Institute - Finance Working Paper No. 610/2019.

Lakonishok, J., A. Shleifer, and R.W. Vishny. 1992. The Impact of Institutional Trading on Stock Prices. Journal of Financial Economics 32 (1): 23-43.

Litterman, R. 2015. David Swensen on the Fossil Fuel Divestment Debate. Financial Analysts Journal 71 (3): 11-12.

PDC. 2015. The Portfolio Decarbonization Coalition. Mobilizing Financial Markets to Drive Economic Decarbonization. https:// unepfi.org/pdc/wp-content/uploads/pdc_brochure.pdf. Accessed 5 Sept 2019.

PDC. 2017. Portfolio Investment in a Carbon Constrained World: The Third Annual Progress Report of The Portfolio Decarbonization Coalition. https://unepfi.org/pdc/wp-content/uploads/PDC-Progr ess-Update-2017.pdf. Accessed 6 Sept 2019.

Pompeo, Michael R. 2019. On the U.S. Withdrawal from the Paris Agreement. Press Statement. U.S. Department of State. November, 4. https://www.state.gov/on-the-u-s-withdrawal-from-theparis-agreement/. Accessed 05 Dec 2019.

Popescu, M., and Z. Xu. 2018. Leading the Herd: Evidence From Mutual Funds' Buy and Sell Decisions. Review of Quantitative Finance and Accounting 50 (4): 1131-1146.

Scharfstein, D.S., and J.C. Stein. 1990. Herd Behavior and Investment. The American Economic Review 80 (3): 465-479.

Sias, R.W. 2004. Institutional Herding. The Review of Financial Studies 17 (1): 165-206.

Springer Nature. 2016. Donald Trump's Choice for Head of the US Environment Agency is Dismaying. 540:315-316

Thomson Reuters. 2015. Asset4 ESG Environmental Datatypes. http:// lipas.uwasa.fi/ jaty/thomson/ASSET4_ESG_Content_Datastream _Premium_Datatypes.xlsx.
Trinks, A., B. Scholtens, M. Mulder, and L. Dam. 2017. Divesting Fossil Fuels: The Implications for Investment Portfolios. Groningen: University of Groningen. SOM research school. SOM Research Reports.

Wermers, R. 1999. Mutual Fund Herding and the Impact on Stock Prices. The Journal of Finance 54 (2): 581-622.

Worley, W. 2016. Marrakesh Climate Conference: Campaigners React With 'Extreme Disappointment' Over Lack of Progress. https:// www.independent.co.uk/environment/marrakech-climate-confe rence-cop-22-campaigners-extreme-disappointment-a7426426. html.

Wylie, S. 2005. Fund Manager Herding: A Test of the Accuracy of Empirical Results Using U.K. Data. The Journal of Business 78 (1): $381-403$

Publisher's Note Springer Nature remains neutral with regard to jurisdictional claims in published maps and institutional affiliations.

Lukas Benz graduated in business administration from the University of Augsburg, Germany, in 2016. In 2016, he joined the Chair of Finance and Banking at the same university as a doctoral candidate. His research activities are focused on mutual funds and green finance.

Andrea Jacob graduated in business administration from the University of Augsburg, Germany, in 2016. In 2017, she joined the Chair of Finance and Banking at the same University as a doctoral candidate. Her research activities are focused on empirical asset pricing and green finance.

Stefan Paulus graduated in business administration from the University of Augsburg, Germany, in 2015. In 2015, he joined the Chair of Finance and Banking at the same university as a doctoral candidate. His research activities are focused on green finance.

Marco Wilkens graduated in business administration from the University of Hamburg, Germany, in 1988. Then, he joined the Chair of Banking and Insurance at the same university as a doctoral candidate. $\mathrm{He}$ received the doctoral degree in business administration from the University of Hamburg in 1993. From 1993, he was a postdoctoral research associate at the Chair of Banking and Finance at the University of Göttingen, Germany. He received his habilitation from the same University in 1999. From 2001 to 2010, he was Professor of Finance and Banking and holder of the Chair of Finance and Banking at the Catholic University of Eichstätt-Ingolstadt, Germany. Since 2010, he has been Professor of Finance and Banking and holder of the Chair of Finance and Banking at the University of Augsburg, Germany. He has been a visiting Professor of Finance and Banking at the University of New South Wales, the University of Sydney, and at the Macquarie University, Australia, as well as at the University of Tasmania. His research activities are focused on mutual funds, interest rate risk, credit risk, carbon risk, risk management, sustainable finance, bank management, valuation of financial instruments, and financial engineering. 